\title{
A Comparative Evaluation of Anti-Tumour Activity Following Oral And Intravenous Delivery of Doxorubicin In A Xenograft Model of Breast Tumour
}

\author{
Farah Rehan \\ Monash University Malaysia \\ Md. Emranul Karim \\ Monash University Malaysia \\ Nafees Ahemad \\ Monash University Malaysia \\ Mohd. Farooq Shaikh \\ Monash University Malaysia \\ Manish Gupta \\ Monash University Malaysia \\ Siew Hua Gan \\ Monash University Malaysia \\ Ezharul Hoque Chowdhury ( $\nabla$ md.ezharul.hoque@monash.edu ) \\ Monash University Malaysia
}

\section{Research Article}

Keywords: biodegradable, drug delivery, nanomicelles, Doxorubicin

Posted Date: August 11th, 2021

DOI: https://doi.org/10.21203/rs.3.rs-775257/v1

License: @ (i) This work is licensed under a Creative Commons Attribution 4.0 International License. Read Full License 


\section{Abstract}

Extensive studies have been conducted on natural materials due to their unique and biodegradable properties for oral drug delivery. In the current research, we fabricated sodium caseinate nanomicelles ( $\mathrm{NaCNs}$ ) using casein as a natural polymer to develop a controlled-release oral drug delivery system. NaCNs was explored to improve the therapeutic potential of Doxorubicin (DOX) with reduced toxicity in healthy organs. The synthesized and thoroughly characterized DOX-loaded NaCNs were subjected to in vivo anti-tumour evaluation and bio-distribution analysis in a 4T1-induced breast cancer model. Our findings indicated an almost eight-fold reduction in tumour size in the group orally treated with DOX-NaCNs when compared to free DOX. It is plausible that the enhanced anti-tumour effects of oral DOX-NaCNs is related to the controlled release of DOX from the delivery system as well as the longer circulatory time of the drug in the blood, as confirmed by the bio-distribution study, when compared with free DOX and the intravenous formulation of DOX-NaCNs. Additionally, the tumour drug accumulation for the orally administered DOXNaCNs was 1.27- and 6.8-fold higher than that of the intravenously administered DOX-NaCNs and free DOX, respectively and overall, was 8.34-times higher than the orally administered free DOX. In comparison, the orally administered DOX-NaCNs showed significant reduction in tumour size $\left(5.66 \pm 4.36 \mathrm{~mm}^{3}\right)$ after the two doses as compared to intravenously administered DOX-NaCNs $\left(10.29 \pm 4.86 \mathrm{~mm}^{3}\right)$ on day 17 of the experimental period. Moreover, $\mathrm{NaCNs}$ were well tolerated in the mice at a single dose of 2,000 mg/ $\mathrm{kg}$ in an acute oral toxicity study. Moreover, no significant change in the body weight was observed after treatment with the single dose of NaCNs when compared to the control group. Thus, NaCNs emerge as a safe and non-toxic delivery system with excellent biodistribution profile and high anti-tumour effects that present as a potential option for oral chemotherapy.

\section{Introduction}

Tumour-targeted delivery of chemotherapeutic agents aimed at enhancing drug concentrations inside the tumour and simultaneously reduce their side-effects while promoting patient compliance with chemotherapy has been an important issue and area of focus in the drug delivery field [1]. For effective chemotherapy, the uptake of chemotherapeutic agents by non-target cells and their quick clearance by kidneys must be minimized, which in turn, can contribute to prolonging blood circulation of these agents and accelerating their accumulation in solid tumours [2].

Following Paul Ehrlich's idea of a "magic bullet", the parenteral route for delivery of anti-cancer drugs is generally preferred to oral, in order to deliver drugs directly to targeted sites rather than to healthy tissues [3]. However, intravenous (IV) administration of various chemotherapeutic agents may cause some distress and discomfort to patients and is confirmed to be an economical burden on ordinary people diagnosed with cancer. This phenomenon is due to the fact that multiple hospitalisations are required to complete the relatively long IV sessions of a combined chemotherapeutic regimen [4].

In the $21^{\text {st }}$ century, oral chemotherapy becomes a vital topic to change the current chemotherapy regimen and the patients quality of life radically [5]. To date, various colloidal drug carriers are being investigated for various routes including the oral method [3]. However, the key challenges of oral delivery include poor drug solubility, drug stability at various pHs of the gastrointestinal (GI) tract, the presence of digestive enzymes that can degrade drugs as well as the protective mucus layer that can block drug penetration across the epithelium and the subsequent absorption into the blood [6]. Another significant factor contributing to the GI barrier is the existence of multidrug efflux proteins, i.e. Ptype glycoproteins (P-gp) found mainly in the epithelial membranes of the GI tract. Some researchers employed P-gp inhibitors, such as cyclosporine A to circumvent this problem; however, they could suppress the body's immune system and cause further medical complications [5]. Thus, designing and formulating an oral dosage form for an 
anticancer agent requires several carefully considered strategies to ensure good bioavailability with reduced sideeffects. Inhibiting or minimizing drug release in the stomach and small intestine [7] is also an added advantage.

Due to the recent advancement in nanotechnology, various approaches are taken based on multi-functional nanoparticles in order to address the limitations involved in prevention, earlier detection and creating effective cancer treatments as well as to significantly enhance the comfort of cancer patients [8-10]. In this respect, nanoparticles may be harnessed as drug carriers for improved oral delivery of various drugs by 1) enhancing the solubility of hydrophobic drugs in the aqueous environment of the $\mathrm{Gl}$ tract 2) preventing premature degradation of unstable drugs and 3) facilitating sustained drug absorption from mucus-lined epithelium into the blood via trans- or paracellular routes $[11,12]$. Once drug-loaded particles are in the blood circulation, the relatively large size of the resultant complexes compared to the free drugs will confer additional advantages, akin to an intravenously administered nanoformulation. This phenomenon occurs due to the increased plasma half-life of the encapsulated drug by restricting broad tissue distribution (such as non-target cells) and thus accelerating tumour accumulation through the 'enhanced permeability and retention' (EPR) effect [13, 14].

Various polymeric nano carriers and core-forming blocks of polymeric micelles such as pluronics, poly(esters) like poly(lactic acid) (PLA), hydrophobic poly(amino acids), copolymers of lactic and glycolic acids as well as poly (caprolactone) (PCL) have been extensively investigated in the past years for their oral uses [15]. For instance, amphiphilic block copolymers consisted of a micellar shell-forming poly(ethylene glycol) (PEG) and a core-forming poly(2-(4-vinylbenzyloxy)-N,N-diethylnicotinamide) (P(VBODENA)) block. Herein, N,N-Diethylnicotinamide (DENA) in the micellar inner core improved Paclitaxel solubilization and stabilization against colon cancer lines with oral bioavailability recorded as $12.4 \%$ of that of the intravenous administration $[5,16]$. On the other hand, D-a-tocopherol polyethylene glycol 1000 succinate (TPGS) can improve the intestinal absorption of drugs such as teniposide and increased teniposide concentration over 7-fold in tumour-bearing mice [17]. In addition, polymeric micelles based on monomethylether poly(ethyleneglycol)(750)-poly(caprolactone-co-trimethylene carbonate) (mmePEG750P) also showed very low clearance by the reticuloendothelial system (RES) and renal excretion. Further studies confirmed that the polymer did not affect P-gp pumps $[18,19]$ indicating its potential.

Natural polymers such as the major milk protein casein have recently attained great attention for drug delivery due to their low cost, easy availability, biodegradability, non-toxicity, unique structural and physicochemical properties as well as the ability to form micelles like a di-block copolymer [20-24]. Although casein micelles re-assembled from sodium caseinate can be used as natural casein micelles, the distinguishing feature of synthetic casein micelles over the natural ones is the tailor-made particle size. Furthermore, drug entrapment is performed before the re-assembling process, which confers higher encapsulation efficiency [25].

In the past, casein-based micelles and nanoparticles were employed to encapsulate folic acid [22], resveratrol [26], vitamin D [27], paclitaxel [28], curcumin [29, 30], thymol [31] and mequindox [32] for oral delivery. The major factor contributing to increased oral bioavailability is the controlled release of drugs from the casein molecules in the intestinal environment, allowing sufficient uptake of these agents from the intestine. There are two probabilities 1) controlled release of the encapsulated drug from the casein micelle in the small intestine resulting in the controlled absorption of the free drug through the epithelium into the blood [26] although this process may not increase drug accumulation in the tumour compared to the orally or intravenously administered free drug and 2) absorption of drugloaded micelles into the blood which increases the drug's plasma half-life by reducing drug distribution to off-target organs and renal clearance, thereby enhancing tumour-selective delivery via EPR effect [33]. Normally, dugs are encapsulated inside the casein molecules through physical embedding, electrostatic interactions and covalent bonding [24]. 
Doxorubicin (DOX) belongs to anthracycline antibiotic and is considered as one of the most effective chemotherapeutics currently being used to treat various cancers, including leukemia, sarcomas, solid tumour of breast, ovaries or thyroid, non-Hodgkin's and Hodgkin's lymphomas. DOX exerts its cytotoxic effects mainly through inhibition of topoisomerase II, DNA double helix intercalation, production of reactive oxygen, species (ROS), activation of caspases, mitochondrial dysfunction and induction of p53 and activation of caspases. Although it has a shorter half-life, drug resistance development and severe cardiotoxicity have particularly limited its clinical use [34]. The FDA however has approved various DOX-loaded nano-formulations, including Abraxane, DaunoXome and Doxil/Caelyx, for clinical use in malignant cancer. To date, some nano-formulations of DOX are currently under clinical investigations, including Aurimmune (phase I), Genexol-PM (phase I) and ThermoDox (phase III). Nevertheless, the low stability in physiological conditions and systemic toxicity hinder the wide applications of liposome- or macromolecules-based nano-formulations for cancer treatment [35].

In this study, we report on the fabrication and characterization of DOX-loaded sodium caseinate nanomicelles (NaCNs) and evaluated the biodistribution profile and therapeutic potential of the resultant complex in a syngeneic mouse model of breast cancer following oral administration, in comparison to the intravenously delivered DOX$\mathrm{NaCNs}$ and the free drug (DOX). DOX-NaCNs were characterized through Zetasizer, field emission scanning electron microscope (FESEM), transmission electron microscopes (TEM) and fourier-transform infrared spectroscopy (FTIR).

In vitro and in vivo evaluations demonstrated robust anti-cancer efficacy of DOX-NaCNs when compared to the free drug alone. Orally delivered DOX-NaCNs significantly reduced tumour burden and restricted the accumulation of DOX to other organs like heart and clearance through RES organs as compared to intravenous DOX-NaCNs and free DOX, suggesting the unique potential of DOX-NaCNs for oral delivery against breast cancer. To our knowledge, this is the first comprehensive study demonstrating the bio-distribution profiles and therapeutic potential of DOX-NaCNs for both intravenous and oral administrations.

\section{Materials And Method}

\subsection{Materials}

Sodium caseinate from bovine milk and DOX (10 mg) and acetone was purchased from the Sigma-Aldrich, Germany. Dulbecco's Modified Eagle Medium (DMEM) was from Nacalai tesque inc, Japan, Fetal Bovine Serum (FBS) and penicillin-streptomycin (P/S) were from Gibco, life technologies, U.K., while 3-(4,5-dimethyl thiazolyl-2)-2,5diphenyltetrazolium bromide (MTT) was from Merck, Malaysia and trypsin-ethylene diamine tetra-acetate (trypsinEDTA) salts were obtained from Sigma-Aldrich. MCF-7 and MDA-MB-231 cell lines were from ATCC (Manassas Va, USA).

\subsection{Methods}

\subsubsection{Preparation and Synthesis of DOX-loaded NaCNs}

NaCNs were formulated by a gentle mixing on a nutating mixer at room temperature, as previously reported [36]. DOX was used a model drug in the current research to evaluate the efficacy of hydrophilic drug inside NaCNs. Briefly, DOX in water solution $(10 \mu \mathrm{M})$ was added (up to $1 \mathrm{~mL}$ ) to the sodium caseinate powder $(1 \mathrm{mg})$ to form the DOX-NaCNS micelles on nutating mixer by vortexing for $1 \mathrm{~h}$. NaCNs re-assembled to load DOX, since caseinate has many amphiphilic phosphate subunits that can form micelles [33]. Along with the DOX-NaCNs, negative control (containing only DOX in water) and the blank NaCNs without DOX were also prepared under similar conditions as described above. 


\subsubsection{DOX-loading into micelles}

Loading efficiency (L.E.) is an essential physicochemical characteristic to evaluate the entrapment of drug in casein nanomicelles The percentage drug loading efficiency (DLE) and percent drug loading content (DLC) of DOX-NaCNs were measured via an indirect centrifugation method [37]. The samples were centrifuged for 20 minutes at 15,000 rpm at $4^{\circ} \mathrm{C}$ by using a centrifuge (Eppendorf Centrifuge $5424 \mathrm{R}$, Sigma Aldrich, Germany). The concentration of free drug was determined by carefully collecting the supernatant from the centrifuge tubes. Subsequently, the absorbance was determined by using a fluorescence spectrophotometer (Glomax Explorer GM3500, Promega Corporation Australia) at $475 \mathrm{~nm}$ (excitation wavelength) and $550 \mathrm{~nm}$ (emission wavelength). The DLE and the DLC (w/w) were calculated from equations 1 and 2 as below [38].

$$
\begin{aligned}
& \% D L E=\frac{\text { Mass of drug in nanoparticles }}{\text { Mass of nanoparticles recovered }} \times 100 \\
& \% D L C=\frac{\text { Mass of drug entrapped in nanoparticles }}{\text { Mass of drug used in formulation }} \times 100
\end{aligned}
$$

\subsubsection{Particle size analysis through dynamic light scattering (DLS) and stability test}

Particle size, distribution and the zeta-potential were measured based on the DLS technique using Malvern nano zeta sizer (Malvern, Worcestershire, U.K.) [39]. The formulations were diluted with water (1:10) at an ambient temperature followed by measurement using a Zetasizer. To evaluate the DOX-NaCNs stability, the samples were stored at $4 \mathrm{C}$. Later particle size, distribution and the zeta-potential were measured over a three months period by using a Malvern zeta sizer at $25 \pm 0.1^{\circ} \mathrm{C}$ following a ten times dilution steps. Measurements were taken three times at 12 runs each time and were reported as mean $( \pm S D)$.

\subsubsection{FESEM and HR-TEM imaging of nanomicelles}

The shape, surface morphology and size analyses of the nanomicelles were investigated using FESEM (Hitachi/SU8010, Tokyo, Japan) at $5.0 \mathrm{kV}$ [39]. Samples were prepared as mentioned in the methodology section (2.2.1). Subsequently, $10 \mu \mathrm{l}$ of sample was placed on the glass cover and was left to be air-dried at room temperature. Before FESEM sample analysis, dried sample was placed on the carbon tape-coated sample holder, followed by platinum sputtering with $30 \mathrm{~mA}$ sputter current for $40 \mathrm{~s}$ at 2.30 tooling factor. Both blank and drug-loaded samples were observed under FESEM. For morphological analysis via HR-TEM imaging, the samples were prepared by suspending the copper grid (300 mesh size) in the nanomicelles suspension. The grid was then dried at room temperature and was analysed using a HR-TEM (FEI tecnai G2 20S-TWIN, Netherlands) at 200 kV.

\subsubsection{Compatibility study of DOX-NaCNs using FTIR}

FTIR enables identification of chemical bonds in drug molecules, polymers or proteins used in formulations [40]. The IR spectra of sodium caseinate, $\mathrm{DOX}, \mathrm{NaCN}$ and $\mathrm{DOX}-\mathrm{NaCN}$ and their respective physical mixture were recorded using a Varian FTIR equipped with a Varian Resolution Pro 640 software (Agilent, Santa Clara, CA, USA). The infrared (I.R.) spectra were taken over the range of $4000-500 \mathrm{~cm}^{-1}$.

\subsubsection{In vitro drug release profile}


To assess the pattern of drug release from DOX-NaCNs at different pHs (7.4/5.0), an in vitro release study was conducted through a dynamic dialysis method modified from previously reported one [37]. DOX-NaCNS were prepared and then the sample $(1 \mathrm{mg} / \mathrm{mL})$ was sealed inside the dialysis membrane. Prior to use, the membrane was activated and soaked overnight inside ultra-pure water. Subsequently, the dialysis bag was tied to the paddle of USP XXIV dissolution apparatus II, apparatus (Electro lab dissolution Tester USP, TDT-08L, India) and was dialyzed against 250 $\mathrm{mL}$ of phosphate-buffered saline $(\mathrm{pH} 7.4 / 5.0)$. The entire system was maintained at $37 \pm 2^{\circ} \mathrm{C}$ under a continuous magnetic stirring (100 rpm) for 24 hours. At the same time, the free drug solution was also dialyzed using a similar dissolution media. The medium was withdrawn $(1.5 \mathrm{~mL})$ at specific time intervals and was replaced with a fresh buffer to maintain a constant volume and sink condition. DOX content in the samples was then measured at $475 \mathrm{~nm}$ (excitation wavelength) and $550 \mathrm{~nm}$ (emission wavelength) using a fluorescence spectrophotometer.

\subsubsection{In vitro cell viability and cytotoxicity studies}

\subsubsection{Cell culture and seeding}

MCF-7 and MDA-MB-231 are human breast cancer cell lines used in the current research to determine in-vitro cell viability and cytotoxicity. Both cell lines were cultured in $25 \mathrm{~cm}^{3}$ flask with a complete DMEM containing $10 \%$ FBS and $1 \% \mathrm{P} / \mathrm{S}$ antibiotic. The flask was then placed in a humidified incubator at $37^{\circ} \mathrm{C}$. The growth medium was subjected to change on every alternate day after an $80 \%$ confluency is achieved. Once confluent, the cells were trypsinized with $0.05 \%$ of trypsin (Sigma, USA) and then passaged in $75 \mathrm{~cm}^{2}$ tissue culture flasks. The cells were then allowed to grow in 96-well containing 50,000 cells per $\mathrm{mL}$ for $24 \mathrm{~h}$ before drug treatment.

The cell viability and cytotoxicity were measured using an MTT assay [41, 42]. After $24 \mathrm{~h}$ of cell seeding, the cells were treated with different concentrations of free DOX (0.0625-1.0000 $\mu \mathrm{M})$. DOX-loaded NaCNs were prepared in the presence of similar drug concentrations, along with blank NaCNs and control (only media) for another $48 \mathrm{~h}$. Following treatment, MTT stock solution [ $5 \mathrm{mg} / \mathrm{mL}$ in phosphate buffer solution (PBS)] was added into each well. After $4 \mathrm{~h}$ of incubation, $100 \mu \mathrm{L}$ of dimethyl sulphoxide (DMSO) was added into each well to dissolve the purple formazan crystals. Subsequently, the absorbance was measured at $560 \mathrm{~nm}$ against a $600 \mathrm{~nm}$ reference wavelength by using a microplate reader (Glomax Explorer GM3500, Promega Corporation Australia) with a built-in plate shaker to shake the plates for 10 secs before measuring the absorbance.

The percentage cell viability (\% CV) was calculated by using a formula:

\section{$\%$ Cell Viability $(C V)=\frac{\text { Absorbance of treated cells-Absorbance of refrence }}{\text { Absorbance of Control--Absorbance of refrence }}$}

where the concentration causing $50 \%$ inhibition $\left(\mathrm{IC}_{50}\right)$ of the free and bounded DOX was also calculated using a Graph pad prism (version 8.0). All the experiments were performed in triplicates and the standard deviations (S.D.) were calculated for mean values.

\subsubsection{Cellular uptake}

\subsubsection{Qualitative analysis}

Qualitative cellular uptake of DOX, a fluorescent compound, was measured using a fluorescence microscope [43, 44]. MCF-7 cells were seeded on a 24 well plate with a density of 50,000 cells per well followed by an incubation step for $24 \mathrm{~h}$. After the incubation, the medium was replaced with $10 \mu \mathrm{M}$ and the $5 \mu \mathrm{M}$ of free DOX and DOX-NaCNs 
respectively prepared in the presence of similar drug concentrations (10 and $5 \mu \mathrm{M})$. The control was prepared by treating the cells with DMEM. The treated cells were incubated for 4 and $24 \mathrm{hr}$. After removing the media, the cells were treated with ethylenediaminetetraacetic acid (EDTA) (5 mM) in PBS to remove the extracellular particles followed by visualisation under a fluorescence microscope.

\subsubsection{Quantitative analysis using a fluorospectrophotometer}

A quantitative cellular uptake analysis was conducted after the treated cells were washed with PBS several times followed by lysis using a lysis buffer. Cell lysate $(200 \mu \mathrm{L})$ was collected from the 24 well plate and was subjected to a fluorescence intensity measurement using a fluoro-spectrophotometer (PerkinElmer, USA) coupled with a 2030 manager software attached with 2030 multilabel reader victor (X5) with an excitation at $485 \mathrm{~nm}$ and an emission at $535 \mathrm{~nm}$. The fluorescence intensity of cells was measured to determine the relative amount of the DOX successfully internalized by cells.

\subsubsection{In vivo tumour regression study}

\subsubsection{Animals}

Female healthy Balb/c mice (5-6 weeks old) were selected and randomized with body weights. The animals were procured from Monash University Malaysia animal facility. The mice were acclimatised for seven days prior to the study. The mice were maintained under a standard husbandry, stress-free and non-pathogenic conditions with a $12: 12 \mathrm{~h}$ light and dark cycle, at $25 \pm 2^{\circ} \mathrm{C}$ and relative humidity of $50 \pm 10 \%$. Water and food were given ad libitum. The study was approved by Monash University Malaysia Animal Ethics Committee (Project ID: 2020-19843-39399) following standard protocols for animal handling and care.

\subsubsection{Synthesis of DOX-loaded micelles}

The formulations were also fabricated for comparative analysis with the blank NaCNs, where DOX-loaded NaCNs were prepared by adding an aqueous solution (up to $1 \mathrm{~mL}$ ) of DOX (at an equivalent dose of $5 \mathrm{mg} / \mathrm{kg}$ ) to sodium caseinate $(1 \mathrm{mg})$ in order to formulate the micelles through nutating mixer by vortexing.

\subsubsection{Induction of murine breast tumour}

Murine breast cancer cells (derived 4T1 cells) were cultured in the complete media (DMEM) containing 1\% P/S antibiotic and $10 \%$ FBS in a $25-\mathrm{cm}^{2}$ flask and was subsequently placed in a humidified incubator (at $37 \mathrm{C}^{\circ}$ and $5 \%$ carbon dioxide). The cells were further sub-cultured in a $75-\mathrm{cm}^{2}$ flask and then trypsinised, once the cells reached the exponential growth phase. After counting the number of cells using a hemocytometer, the cells were re-suspended in DMEM at $10^{6}$ cells $/ \mathrm{mL}$ and were later suspended in PBS to yield a concentration of $10^{5} \mathrm{cells} / 100 \mu \mathrm{L}$.

On day 1 , the cells were subcutaneously injected into the left side of the mice's mammary gland (at $1 \times 10^{5}$ cells $/ 100$ $\mu \mathrm{L}$ ) using a $27 \mathrm{G}$ needle. The mice which developed tumours were observed at least three times weekly until the presence of a palpable tumour nodule, following which daily monitoring was conducted. One to two weeks following the inoculation, when the tumours reached an average volume of $13.73 \pm 2.51 \mathrm{~mm}^{3}$, the mice were randomly assigned to the various treatment groups in the study ( $n=5 /$ group). Following the injection of the samples, the size of the tumour (volume) was monitored at regular intervals from days 1 to 28 , with the help of a digital Vernier calliper.

The below formula was used to measure tumour volume: 
Tumour Volume $\left(\mathrm{mm}^{3}\right)=\frac{1}{2}\left(\right.$ lenght $\times$ width $\left.{ }^{2}\right)$

\subsubsection{In-vivo anti-tumour effects}

To evaluate the anti-tumour effect, the animals were divided into eight groups ( $\mathrm{n}=5$ for each group). Tumour-induced mice were administered with free DOX solution, DOX-NaCNs formulation ( $5 \mathrm{mg} / \mathrm{kg} / \mathrm{day})$ along with the negative control and Blank NaCNs both intravenously and orally. Intravenous administration was done into either the right or left side of the tail vein via a $29 \mathrm{G}$ needle. Oral administration is via a steel oral gavage $(11 \mathrm{G})$ via the oesophagus into the stomach with a gap of $48 \mathrm{~h}$ for comparative analysis between micelles being administered intravenously and orally. Treatment was given on days 14 and 17. The weight of the mice was determined at regular intervals for every three days.

At the end of the experiment (day 28), the mice were exposed to 100\% carbon dioxide in a chamber for euthanisation. Subsequently, the vital organs including the brain, spleen, lungs, liver, kidney and heart as well as any visible tumours were removed. The excised tumour and different organs of the mice were washed in cold PBS and the weight determined. All the measurements were presented as mean \pm SD for each group.

\subsubsection{Biodistribution of intravenously and orally administered DOX-loaded micelles in a xenograft mouse model}

The $4 \mathrm{~T} 1$ cells $\left(1 \times 10^{5}\right.$ cells $\left./ 100 \mu \mathrm{l}\right)$ in PBS were subcutaneously injected into the mammary pads of female balb/c mice (5-6 weeks of age) weighing 17-19 g for breast tumour induction. Tumour-bearing animals were divided into two sets, where one set of mice (three groups; $n=4$ /group) were orally treated with NaCNs, free DOX and DOX-NaCNs using a steel gavage. In contrast, the other set (three groups; $n=4$ /group) were intravenously treated with $\mathrm{NaCNs,} \mathrm{free}$ DOX and DOX-NaCNs via the caudal tail vein. An equivalent dose ( $5 \mathrm{mg} / \mathrm{kg}$ ) was used to prepare free DOX and DOX$\mathrm{NaCNs}$. Blank NaCNs were used as controls in both sets of experiments. One day post-administration of the formulations, the mice were anaesthetized with an appropriate dose of ketamine/xylazine calculated based on the body weight before blood collection into heparinised tubes via cardiac puncture. Subsequently, the animals were sacrificed through cervical dislocation before collection of vital organs (brain, heart, spleen, liver, lung and kidney) and tumour for the treated mice. All the organs were kept in the $400 \mu \mathrm{L}$ of lysis buffer $(\mathrm{pH} 7.4)$ after being washed with PBS and were stored at $-150^{\circ} \mathrm{C}$ for further analysis.

The organs were mechanically homogenized and centrifuged at $8000 \mathrm{rpm}$ for $20 \mathrm{~min}$ at $4^{\circ} \mathrm{C}$. The supernatant (100 $\mu \mathrm{L}$ ) was collected and placed in a 96 well plate to determine the fluorescence intensity of DOX at $475 \mathrm{~nm}$ (excitation wavelength) and $550 \mathrm{~nm}$ (emission wavelength) using a fluorescence spectrophotometer. The data was presented as relative means of the values \pm SD (after being blank corrected from the control group values) in fluorescence intensity unit/mg of the tissue mass.

\subsubsection{Blood analysis}

Blood serum analysis also conducted following blood collection from all groups via a cardiac puncture using $27 \mathrm{G}$ needle. The samples were centrifuged at $10,000 \mathrm{rpm}$ for 30 mins at $4^{\circ} \mathrm{C}$ to collect the supernatant (blood serum) and the fluorescence intensity of the samples was calculated using a fluorescence spectrophotometer (PerkinElmer, USA. 2030 manager software attached with a2030 multilabel reader victor X5) at 485/535 nm.

\subsubsection{Toxicity evaluation of micelles and determination of LD50}

\subsubsection{Acute oral toxicity study}


The Organization for Economic Co-operation and Development (OECD) 425 up-and-down procedure [45] is often used to minimize the number of animals required in acute oral toxicity testing. The current study is based on the OECD 425 up-and-down guideline to determine the $50 \%$ lethal dose $\left(\mathrm{LD}_{50}\right)$ of $\mathrm{NaCNs}$. The changes in the mice's behaviour were also monitored according to the Irwin test [46] to observe the overall health and well-being of mice.

\subsubsection{Toxicity profile}

Balb/C mice were selected for toxicity studies based on the micelles' evaluation of against breast cancer cells following loading with anti-cancer drugs. Furthermore, it was selected following their various attributes including having a long span of life, showing resistance against atherosclerosis development, due to their simple dietary issues and also due to ease of monoclonal antibodies production. The mice were divided into two groups; control (water) and treated (blank NaCNs), with five animals per group.

On the first day, one of the mice was administered with a single dose of casein micelles (2000 mg/kg) through oral gavaging. The short time toxicity profile was observed at different points (0, 15, $30 \mathrm{~min}, 1,2,4$ and $24 \mathrm{~h})$. Any clinical sign of toxicity/abnormality such as lower food or water intake or any signs of morbidity and mortality among the treated mice was recorded. Based on the short-term toxicity profile $(24 \mathrm{~h})$ of the mouse, a similar dose $(2000 \mathrm{mg} / \mathrm{kg})$ was simultaneously administered to another four mice and the short-term toxicity parameters were observed. The mice were kept under observation for 14 days to establish any further toxic signs.

The overnight-fasted mice were administered with a single dose of micelle formulation $(2000 \mathrm{mg} / \mathrm{kg}) \mathrm{by}$ a an oral gavage needle $(11 \mathrm{G})$. Some of the critical short term toxicity parameters recorded based on Irwin's test include:

i. Righting Reflex (R.R.)

The ability of the mouse to regain healthy posture within 30 secs after being placed flat on its back.

ii. Body Weight

Abnormality in body weight was observed where a $15 \%$ change in body weight is deemed as a sign of toxicity.

iii. Sedation

Reduction in the movement of the animal indicates a sedative effect even after manipulation.

iv. Clinical Signs

Abnormalities in the frequency of urination and defecation were monitored.

After 14 days of observation, the mice were weighed and euthanized [47] before further analysis.

\subsubsection{Clinical observations}

Following a single dose administration of $\mathrm{NaCNs}(2000 \mathrm{mg} / \mathrm{kg})$, the mice were monitored continuously at regular intervals $(0,15,30 \mathrm{~min}, 1,2,4$ and $24 \mathrm{~h}$ ) and then for 14 days. All animals (both control and treated groups) were observed twice daily for body posture changes, general appearance, condition of the skin, eyes, nose, oral cavity, fur, eyes, nose, oral cavity, abdomen as well as morbidity and mortality signs as per Irwin's test. All the parameters were recorded once before treatment and then twice daily subsequently. In addition, body weight, water and food 
consumption were measured and recorded every other day throughout the study period, before necropsy. All animals survived with no mortality seen till the end of the observation period of almost two weeks.

\subsubsection{Statistical analysis}

Statistical analyses of the in vitro and in vivo studies were performed using Graph pad prism version V8. For the pairwise comparison analysis, ANOVA and Turkey's Multiple Comparison tests were used. Values at $p<0.0001$ were considered as highly significant, with a $95 \% \mathrm{Cl}$ (confidence interval).

\section{Results And Discussion}

\subsection{Synthesis and physicochemical characterization of DOX-NaCNs}

$\mathrm{NaCN}$ were prepared by allowing caseins in aqueous solution to self-assemble into micelles $[39,48]$. An aqueous solution of DOX was added to sodium caseinate powder $(1 \mathrm{mg})$ prepared from raw skim milk [49]. The mixture was immediately subjected to vigorous uniform mixing through vortexing to allow caseinates to assemble into micelles while encapsulating DOX in the interior upon placement on the nutating mixing (24 rpm) for $1 \mathrm{~h}$ at an ambient temperature. Blank micelles were prepared similarly without the addition of the drug (DOX). Both DOX-loaded NaCNs and empty NaCNs were characterised in terms of zeta size, polydispersity index (PDI) and zeta potential (Table 1). In addition, drug loading contents (DLC) and drug loading efficiency (DLE) for DOX-NaCNs were measured (Table 1).

Table 1: Zeta size, PDI and zeta potential of blank micelle (NaCNs) and DOX-NaCNs along with DLE and DLC for DOX$\mathrm{NaCNs}$

\begin{tabular}{|lllllllllll|}
\hline Formulations & $\begin{array}{l}\text { Zeta } \\
\text { Size }\end{array}$ & \pm SD & PDI & \pm SD & $\begin{array}{l}\text { Zeta } \\
\text { potential }\end{array}$ & \pm SD & $\begin{array}{l}\text { DLE } \\
(\%) \\
\text { V/v }\end{array}$ & \pm SD & $\begin{array}{l}\text { DLC } \\
(\%) \\
\text { w/w }\end{array}$ & $\pm S$ D \\
Blank NaCNs & 470.20 & 68.12 & 0.471 & 0.009 & -1.93 & 0.53 & - & & & \\
DOX-NaCNs & 270.860 & 17.958 & 0.681 & 0.069 & -0.054 & 0.003 & 78.990 & 1.590 & 24.830 & 1.630 \\
\hline
\end{tabular}

The hydrodynamic size, PDI and morphology are important parameters since they may influence the properties of nanocarriers such as assay design, delivery and migration of NPs as well as their bioconjugates in living tissues and cells. Therefore, characterization of NPs surface charge and hydrodynamic size are required to control bioconjugation of NPs ligand chemistry and performance in biological imaging or assays.

The zeta size measurement through dynamic light scattering (DLS) reveal that the blank NaCNs had a particle size of around $470.2 \pm 68.12 \mathrm{~nm}$. Nevertheless, following DOX encapsulation, the particle size was $270.86 \mathrm{~nm}$, indicating the self-assembled nature of casein [39] Furthermore, interactions of DOX within the micellar interior components of casseinate may reorganize and condense particles into smaller micelles which have a more heterogeneous size distribution and are less electronegative when compared to the empty micelles (Table 1). Additionally, DOX-NaCNs showed robust DLE (78.00\%) and DLC of approximately $24.83 \%$ signifying the successful encapsulation of DOX inside the casein. Since casein micelles are natural diblock amphiphilic polymer, they can increase DOX solubility through hydrogen bonds and hydrophobic interactions [50].

The biological fate of nanoparticles depends on their shapes and sizes as the lymph and the vascular systems play vital roles in the clearance and filtering of non-spherical foreign particles [51]. Considering this fact, the fabricated micelles were designed to have spherical shape as demonstrated in FESEM (Fig. 1A, B and C) and HR-TEM (Fig. 2A 
and B) images. The micelles were smaller in size than the ones analysed by DLS, due to the shrinkage of micelles during the dehydration process during samples preparation for HR-TEM and FESEM analyses [51, 52].

FTIR spectroscopy is an important characteristic tool that offers both qualitative and quantitative analyses of formulations [40]. It can also analyze the absorption bands that are useful in determining the conjugation of a drug carrier with the drug [53].

In the IR spectra of DOX-NaCNs (Fig. 3), DOX showed the characteristic peak at 3278.5, thus showing only a slight shifting of peak in DOX-NaCNs from the free DOX peak $\left(3294.160 \mathrm{~cm}^{-1}\right)$, justifying loading of DOX in NaCNs formulation [54]. The characteristic peaks of free DOX appeared at 3329.741 and $1635.703 \mathrm{~cm}^{-1}$ (Fig. 3) indicating $\mathrm{O}-\mathrm{H}$ stretching $[55]$ and $\mathrm{C}=\mathrm{O}[54,56]$ respectively. In contrast, sodium caseinate showed some characteristic peaks at $3030.310,1533.464$ and $1121.4 \mathrm{~cm}^{-1}$ which originate from $\mathrm{N}-\mathrm{H}$ stretching and amide bending vibrations. Sodium caseinate also exhibited a characteristic peak around $1600 \mathrm{~cm}^{-1}$, indicating the presence of $\mathrm{C}=0[57,58]$. There was no significant shifting in $\mathrm{C}=\mathrm{O}$ of DOX around $1632.11 \mathrm{~cm}^{-1}$ in the DOX-NaCNs indicating the absence of chemical bondings between the $C=0$ radicals of the casein micelles and that for DOX [54]. The slight overlapping and shifting of bands in the IR spectra of the DOX-NaCNs indicated the binding of DOX with the protein [56]. Hence, the IR spectra of DOX-NaCNs confirmed the chemical stability of DOX inside the micelles and its interaction with micelles when compared with the spectra of free DOX and blank NaCNs.

Polymeric micelles tend to suffer from a low colloidal stability, especially following drug encapsulation [59]. Based on the concept that colloid stability can be evaluated via stability measurements or by measuring the aggregation rate in dynamic experiments [60], the colloidal stability of DOX-NaCNs was assessed at $4^{\circ} \mathrm{C}$ through zeta potential, particle size and size distribution. After three months of storage, the particle size of DOX-NaCNs was stable and remained in a desired size range (Fig. 4A) with a slight size increase after 60 days. Subsequently, it decreased in size returning to its approximately normal size before storage and at the end of the third month of storage at $4 \mathrm{C}$. Moreover, zeta potential values (Fig. 4C) of DOX-NaCNs remained in negative values, indicating their stability over the period of three months with no significant signs of aggregation in size distribution values (Fig. 4B).

\subsection{In vitro drug release profile}

The drug release from nanocarriers can either be $\mathrm{pH}$-dependent or occur via an enzymatic hydrolysis. Therefore, the drug release kinetics of $\mathrm{DOX}-\mathrm{NaCNs}$ were investigated at $\mathrm{pHs} 5.0$ and 7.0 to determine the $\mathrm{pH}$ sensitive ability of the carriers [53]. In the tumour, the extracellular environment is acidic due to lactic acid production and the fact that endosomal compartments formed after endocytosis of drug-loaded micelle by cancer cells are acidic. Drug release in a low pH environment indicates that DOX is released in both tumour microenvironment and into the cells after endocytosis. In both cases, the released drugs will enter the cytosol and subsequently, the nucleus after penetrating the plasma and endosomal membranes, respectively. On the other hand, drug release at physiological pH suggests that the drug is released into the blood stream even before going to the targeted site [61]. In this study, both control and DOX-NaCNs exhibited comparatively diverse release patterns at both pHs (Fig. 5A \& B). The free DOX solutions followed the burst and continuous release patterns as compared to DOX-NaCNs, where the release was in a more controlled manner.

Moreover, the release of DOX from DOX-NaCNs was approximaely $50 \%$ in $24 \mathrm{~h}(\mathrm{pH} 7.4$ ), but showed a more controlled pattetn at pH 5.0 as compared to the free drug indicating that more than $50 \%$ of the drug is released within 3 hours. Thus, NaCNs can provide a more sustained release of DOX without the burst release effect, hence lowering the doserelated adverse effect [62]. 
Additionally, casein stability is an added advantage at a lower $\mathrm{pH}$ and does not impact the cumulative release of DOX in a complex biological system [63]. A similar release pattern for casein protein was also previously reported where drugs are more uniformly dispersed in micelles before being released out due to diffusion [64-66]. The pH can also influence the ionization of casein and the integrity of the casein micelle to release drugs. A sustained release of casein would suggest that micelles do not disintegrate prematurely to release drug load especially at physiological $\mathrm{pH}(7.4)$, thus promoting controlled release effect in targeted cancer cells [67].

\subsection{In vitro cell viability and cytotoxicity}

The cell viability and toxicity of DOX-NaCNs were analyzed against human breast cancer cells (MCF 7 and MDA-MB 231) after incubating for $48 \mathrm{~h}$ by using an MTT assay. DOX-loaded NaCNs showed considerable cytotoxicity against both cell lines when compared with both free DOX and blank micelles in almost all dosages that ranged from $0.0625-1.0000 \mu \mathrm{M}$ (Fig. 6).

Following a $48 \mathrm{~h}$ incubation, DOX-NaCNs formulation showed the most significant cytotoxicity against MCF-7 (83\%) and MDA-MB 231 (91\%) cells, at $1 \mu \mathrm{M}$ as compared to free DOX. Besides, DOX-NaCNs also showed an IC $\mathrm{I}_{50}$ of approximately $129.3 \mathrm{~nm}$ as compared to the $\mathrm{IC}_{50}$ of free DOX $(151 \mathrm{~nm})$ against MCF-7 cells. On the other hand, DOX$\mathrm{NaCNs}$ showed an $\mathrm{IC}_{50}$ of approximately $103 \mathrm{~nm}$ as compared to the $\mathrm{IC}_{50}$ of free DOX $(116 \mathrm{~nm})$ against MDA-MB 231. A significant cytotoxicity of DOX-NaCNs was seen against breast cancer cells as compared to the free drug possibly as a result of a more enhanced ability of casein micelles to penetrate cancer cells [68]. The process may occur via an energy-dependent manner though a more efficient endocytosis which facilitates accumulation of the drug in the intracellular active site of nuclei, thus enhancing the cytotoxic effect of the drug-loaded $\mathrm{NaCNs}$ as compared to that for the free drug [43]. Furthermore, following cellular internalization, DOX is released from the micelles due to the low $\mathrm{pH}$ in the acidic endosomes or lysosomes as indicated in our drug release profile assay where the controlled release of DOX from micelles was found at an endosomal pH 5.5 [61].

Moreover, free DOX faces multidrug resistance [56] mainly due to P-gp efflux and also passive diffusion [63] which resulted in higher cell viability in both MCF and MDA-MB 231 cell lines as compared to the loaded micelles. Another reason for the enhanced cytotoxicity may be attributed to the slow release of entrapped drug from the micelle matrix (as demonstrated in our drug release studies at a lower $\mathrm{pH}$ ) which facilitates drug efflux from tumour extracellular environment into the cells through a concentration gradient [69]. The in vitro viability of blank micelles was also analyzed by exposing cells to different micelles concentrations $(6.25,12.50,25.00,50.00$ and $100.00 \mu \mathrm{g} / \mathrm{mL})$ to evaluate the effect of different micelles concentrations along with the free and loaded micelles. Following a $48 \mathrm{~h}$ of incubation, the viability of the cells in almost all the blank formulations was close to that of control (media) at a concentration up to $100 \mu \mathrm{g} / \mathrm{mL}$ (Fig. 9), confirming the non-toxic nature of casein [70].

\subsection{Cellular uptake}

Since uptake is highly-dependent on the size and surface charge of particles [54], the cellular uptake of the DOX$\mathrm{NaCN}$ was also analyzed and compared to the free DOX in water following 4 and $24 \mathrm{~h}$ of incubation with the MCF-7 cells. Additionally, the cellular uptake of nanoformulations greatly influences drug delivery as well as therapeutic efficacy [35]. Therefore, it is important to measure cellular uptake both by qualitative and quantitative analyses.

\subsubsection{Qualitative analysis through confocal microscopy}

For the qualitative analysis, the cellular uptake was investigated using a confocal fluorescence microscopy. The images showed bright green fluorescence signals in the cytoplasm of MCF-cells (Fig. 7) indicating that DOX-loaded 
micelle conferred considerable cellular uptake as compared to free dox as confirmed by the vigorous fluorescence intensity in the cells. Based on the particle size and negatively charged surface of NaCNs, the mode of entrance of micelles inside the MCF-7 cells was through caveolae-mediated endocytosis [71, 72].

\subsubsection{Quantitative analysis}

The intracellular uptake of DOX-loaded NaCNs was also quantitatively evaluated using a lysis buffer to get a more accurate data. MCF-7 cells were treated for 4 and $24 \mathrm{~h}$ with DOX-NaCNs prepared in the presence of 5 and $10 \mu \mathrm{M}$ of the drug. DOX-loaded NaCNs showed both dose- and time-dependent cellular uptake. There was a significantly higher cellular uptake of DOX-NaCNs at both 5 and $10 \mu \mathrm{M}$ of the drugs used to prepare the complexes, as compared to free DOX after $4 \mathrm{~h}$ and $24 \mathrm{~h}$ of the treatment (Fig. 8). Overall, our findings supported the notion that DOX is efficiently internalized inside human breast cancer MCF-7 cells through NaCNs and hence can enhance the therapeutic efficacy of DOX and casein is an excellent promising carrier for cancer treatment.

\subsection{In vivo anti-tumour effect of DOX-NaCNs}

The anti-tumour effects of the optimised DOX-NaCNs was evaluated in the murine breast cancer model. Female mice (5-6 weeks) were inoculated with 4T1 cells subcutaneously and were randomly divided into eight groups, each containing five mice.

\subsubsection{In vivo anti-tumour activity of intravenously administered DOX-NaCNs}

The four groups were intravenously treated with two doses of (i) PBS (ii) blank NaCNs (iii) free DOX solution (5 $\mathrm{mg} / \mathrm{kg}$ ) (iv) DOX-NaCNs (prepared with equivalent dose of $5 \mathrm{mg} / \mathrm{kg}$ of doxorubicin on days 14 and 17. All mice were administered with the above-mentioned formulations intravenously through the tail vein using a $29 \mathrm{G}$ needle with a gap of $48 \mathrm{~h}$. The mice injected with PBS, blank NaCNs and free DOX formed large tumours with continuous growth throughout the experimental period (Fig. 9). Moreover, the tumour excised from the groups treated with the PBS, blank $\mathrm{NaCNs}$ and free DOX appeared to have massive growth (Fig. 10\& supplementary Fig. 3). However, the tumour excised from the mice group treated intravenously with DOX-NaCNs tumour demonstrated a comparatively slower growth. Moreover, the tumour was reduced to a significantly lower size after only two doses of DOX-NaCNs, demonstrating that DOX-NaCNs has a potent anti-tumour effect (Fig. 10\&11).

\subsubsection{In vivo anti-tumour activity of orally administered DOX-NaCNs}

To validate the benefits of using an oral route, one set of four groups $(n=5)$ of mice were treated with orally administered saline, drug-free NaCNs, free DOX solution and the DOX-NaCNs at $5 \mathrm{mg} / \mathrm{kg} /$ day via gavaging.

The marked reduction in the tumour volume was recorded (Fig. 11 and supplementary Fig. 3) in case of orally administered DOX-NaCNs on day $17\left(5.66 \pm 4.36 \mathrm{~mm}^{3}\right)$ after the two doses as compared to the intravenously administered DOX-NaCNs $\left(10.29 \pm 4.86 \mathrm{~mm}^{3}\right)$. At the end of the study, a comparably slower growth of the tumour was observed in orally administered DOX-NaCNs $\left(42.80 \mathrm{~mm}^{3}\right)$ and intravenously administered DOX-NaCNs $\left(51.86 \mathrm{~mm}^{3}\right)$ when compared with free DOX solution administered orally and intravenously (372.92 and $438.56 \mathrm{~mm}^{3}$, respectively) (Fig. 11A). Hence, the anticancer activity of DOX is significantly enhanced when 1$)$ loaded onto micelles $(p<0.001)$ and 2) administered via the oral route.

The significant enhancement of the anti-cancer activity of the DOX-NaCNs seen via oral delivery may be attributed to the longer circulation half-life [73] when compared with intravenously administered DOX-NaCNs. The said hypothesis 
was further validated through our biodistribution studies. Moreover, the tumour growth was reduced until day 20, followed by a slow growth rate up to day 28 in both orally and intravenously administered DOX-NaCNs.. At the same time, the free DOX showed an exponential curve in growth, causing sufficient tumour burden in animals [2]. As compared to the free DOX, the growth rate of the blank micelles was delayed, but the later growth was enhanced with no-toxicity towards the tumour. Furthermore, it is plausible that casein molecules enable DOX to reach the gut epithelium surface [26]. It is hypothesized that DOX-NaCNs are absorbed into the blood from the intestine, thus promoting a higher anti-tumour effects due to a more prolonged half-life and increased tumour accumulation occurring via EPR effect.

The successful tumour regression by both DOX-NaCNs may be attributed to the controlled and biphasic releases of DOX through NaCNs as revealed in our dug release assay or due to the rapid and higher internalization of DOX in the DOX-NaCNs as compared to the free DOX. The significant enhancement of anti-cancer activity of the DOX-NaCNs through oral delivery may be attributed to the longer circulation half-life [73] when compared with free DOX (intravenously and/or orally) and intravenously administered DOX-NaCNs groups. Kanwal et al. [74] reported that encapsulation of DOX in the biodegradable and non-toxic delivery system may prevent its degradation, increasing the half-life of drugs in the circulation. Secondly, it is plausible that the natural assembling property of sodium caseinate into nanomicelles facilitates the passive drug delivery to tumour cells through EPR effect [33]. Furthermore, casein molecules enable DOX to reach the gut epithelium surface and also control the release rate for DOX, thus promoting its absorption and oral bioavailability [26] to confer the anti-tumour effects.

Figure 11 showed the average weight of the excised tumour in each group. DOX-NaCNs treated group had a noticeable reduction $(p<0.05)$ in the average tumour weight when compared with other groups thus demonstrating the good anti-tumour effects of the developed natural protein nanomicelles. Furthermore, sodium caseinate nanomicelles also prevent the prematured release of anti-cancer drug in the blood and maximise the drug concentration in the tumour, which resulted in the enhanced therapeutic effect of the loaded nanosystem.

No apparent change in the body weight was observed in the DOX-NaCNs formulations and NaCNs-treated group in both sets (Figs. 13 \& 14A) signifying the safety of the treatment method and the non-toxicity of the delivery system. However, there was a slight decrease in the weight of mice in the free DOX-treated group which may be due to the high cytotoxicity of DOX in the free form. Furthermore, no significant abnormal behaviour and activity of mouse was seen while all mice have survived the treatment.

To further confirm the toxicity of the nanomicelles, the vital organs (heart, brain, liver, lung, kidney and spleen) were removed after the treatment period of all treated groups and weighed. Both orally and intravenously treated DOX$\mathrm{NaCNs}$ groups showed a significant decrease $(p<0.001)$ in the spleen and liver weights as compared to the free DOXtreated group, [Figs. 13 and 14 (B)] thus indicating that NaCNs can prevent mice from suffering the adverse effects of the anti-cancer drug [68]. Furthermore, the liver is of smaller size (Fig. 12B) in orally administered DOX-NaCNs group, where DOX exhibited a remarkable therapeutic efficacy of orally administered DOX-NaCNs, when compared to both intravenously administered DOX-NaCNs and free DOX-treated groups.

\subsubsection{Biodistribution Study in a Xenograft Mouse Model}

In vivo biodistribution of nanoformulations and their mechanisms of biodegradation and excretion define the fate, viability and applicability of such a nano-delivery platform in the practical clinical translation [75]. Furthermore, the biocompatibility of the synthesized nanoformulations at the levels of cell, blood and tissue is always considered crucial for efficient drug delivery [35]. 
Considering the abovementioned facts, biodistribution studies of the DOX-NaCNs and free DOX were performed in Balb/c mice (5-6 weeks old) after intravenous administration at tail vein and through oral gavage an equivalent DOX dose of $5 \mathrm{mg} / \mathrm{kg}$. In order to monitor the formulations biodistribution and also to determine the therapeutic efficacy of the orally administered DOX-NaCNs, a comparative study was carried out by maintaining the same conditions however one set of mice (three groups with 4 mice per group) was orally administered with the NaCNs, free DOX and DOX-NaCNs, while the other set (three groups with 4 mice per group) was intravenously administered with the NaCNs, free DOX and DOX-NaCNs at an equivalent DOX dose of $5 \mathrm{mg} / \mathrm{kg}$. Blank NaCNs was used as control in both set of experiment. Later after $24 \mathrm{~h}$ post-administration of the all the formulations in both sets of experiments, animals were sacrificed to collect blood, organ tissues and tumour from treated mice. The tumour distribution of the DOX-NaCNs administered orally revealed the higher amount (Fig. 15) when compared with other organs after single dose $24 \mathrm{~h}$ post-administration time frame.

Moreover, Fig. 15 indicates that the tumour drug distribution of the orally administered DOX-NaCNs was 1.27 fold, 6.8 and 8.34 fold more than intravenously administered DOX-NaCNs, intravenously administered free DOX and the orally administered free DOX respectively, whereas intravenously administered DOX-NaCNs exhibited the 4.9 fold and 6.65 fold increase over the intravenously administered free DOX and the orally administered free DOX respectively. Interestingly, the orally administered DOX-NaCNs reduced the drug disposition into the heart significantly $(p<0.001)$ when compared to the free DOX and intravenously administered DOX-NaCNs, thus suggesting the potential of DOX$\mathrm{NaCNs}$ to reduce cardiotoxicity caused by DOX.

Figure 15 also showed that negligible amount of DOX-NaCNs administered orally and intravenously was found in the organs like liver, lung, kidney and spleen (RES). The hydrophilic coat of the NaCNs reduced the plasma protein adsorption, decreased surface charge, enhanced hydrophilicity and inhibit the electrostatic and hydrophobic interactions that permit opsonin to be attached to micelles. All these factors explicitly reduced the liver and spleen drug accumulation and thus prevented the RES elimination [2] and eventually caused higher tumour accumulation of bound drugs. Additionally, efficient release of DOX in acidic $\mathrm{pH}$ (tumour microenvironment and intracellular endosomal compartments are acidic) as well as longer circulation time of drug in blood due to blocking wider distribution of bound drug and less non-specific plasma protein binding on micelles prevent their macrophage uptake and enhanced tumour accumulation via EPR effect. The hypothesis was further tested by quantification of DOX in the mice's blood plasma, $24 \mathrm{~h}$ post formulation administration, where free DOX administered orally showed approximately a five-fold lower drug concentration $(p<0.001)$ in the blood as compared to orally administered DOXNaCNS at $24 \mathrm{~h}$. The orally administered DOX-NaCNS represented a 1.4 fold increase in blood plasma concentration as compared to the intravenously administered DOX-NaCNS (Fig. 16). Interestingly, lower brain accumulation of DOX$\mathrm{NaCN}$ seen may be attributed to endocytosis [76] which requires time.

The enhanced plasma level of orally administered DOX-NaCNS may be due to the longer circulation time as a result of increased membrane permeability and inhibition of P-gp efflux pump through nano-channel entrapment of DOX inside $\mathrm{NaCNs}$ [77]. Furthermore, the pH-dependent behaviour of casein micelles contribute towards the controlled release of the orally administered drugs in addition to the fact that the energy-independent penetration of casein through plasma membrane further enhances cellular uptake upon oral administration [23].

Thus, the enhanced therapeutic efficacy of the orally administered DOX-NaCNS may also contribute to the increased accumulation of DOX by tumour-resistant cells, making DOX-NaCNs an ideal formulation for oral delivery for cancer treatment. Oral delivery is more desirable over IV infusions, which may cause hospitalization and contribute to increased cost while exposing immune-compromised patients to infections [78]. Additionally, the formulation is 
desirable since there was no significant change in the organ's weight of mice treated intravenously and/or orally with DOX-NaCNs compared with free DOX and normal mice groups (Fig. 17).

\subsection{Acute oral toxicity study}

In this study, NaCNs treated with an oral dose of $2,000 \mathrm{mg} / \mathrm{kg}$ did not confer any morbidity or toxicity in the animals. Moreover, there was no significant toxicity, behavioural changes (such as increased in breathing, postural changes) or other abnormalities including skin and fur changes, hair loss as well as change in organ weight observed 4, $24 \mathrm{~h}$ after 14 days of experiment. Additionally, there was no significant weight gain/loss or noteworthy necroscopy findings recorded after the two weeks of the experimental period indicating that there was no significant acute toxicity with $\mathrm{NaCNs}$. The $\mathrm{LD}_{50}$ for female BalbC mice is $>2,000 \mathrm{mg} / \mathrm{kg}$.

\subsubsection{Bodyweight, water and food consumption analysis}

There was no apparent weight changes observed among control and NaCNs-treated mice (Fig. 18). All mice showed a regular increase in weight (Fig. 18A). throughout the observation period with no apparent difference in food and water intake (Fig. 18B and C). However, particular deviation in the food and water consumption occurred among both groups, which may be contributed by various factors including stress caused by oral treatment or fighting among animals housed together, the impact of light, noise or may be due to variation in measured values $[79,80]$. Furthermore, there was no significant difference between food and water consumption among both control and $\mathrm{NaCNs}$ treated mice irrespective of the treatment, further indicating the fact that NaCNs is non-toxic [47].

\section{Conclusion}

In the current study, DOX-NaCNs was synthesized and characterized to explore its antitumour effects and to conduct a biodistribution analysis in a murine breast cancer model. When stored at $4 \mathrm{C}$ for three months, the developed DOXNaCNs showed a maximum E.E of $78.99 \pm 1.55 \%$ with a particle size of $270.86 \pm 17.95 \mathrm{~nm}$ and had colloidal stability. Since $\mathrm{NaCNs}$ were well-tolerated in mice when administered at a single dose of $2,000 \mathrm{mg} / \mathrm{kg}$, the safety of NaCNs was further confirmed in an in vivo test. An almost eight-fold reduction in tumour size in the group treated orally with DOX-NaCNs was observed as compared to free DOX, which may be attributed to the controlled release of DOX from the delivery system as well as the longer circulation time of the drug in blood when compared to the free DOX or DOX$\mathrm{NaCNs}$ intravenous formulation. Thus, the developed sodium caseinate nanomicelles can improve drug's bioavailability when administered orally. $\mathrm{NaCNs}$ is a potential emerging oral drug delivery system that needs to be further explored for tumour-directed delivery of drugs while validating the biodistribution patterns of the loaded drugs in various cancer models.

\section{Declarations}

Funding: Not applicable

\section{Conflicts of interest/Competing interests: There is no conflicts of interests}

Availability of data and material: Not applicable

Code availability: Not applicable

Ethics approval: Animal ethics approval was granted. 
Consent to participate: Not applicable

\section{Consent for publication: All authors gave their consent to publication of the data}

\section{References}

1. Reddy, L.H., R. Sharma, and R. Murthy, Enhanced tumour uptake of doxorubicin loaded poly (butyl cyanoacrylate) nanoparticles in mice bearing Dalton's lymphoma tumour. Journal of drug targeting, 2004. 12(7): p. 443-451.

2. Kim, D., et al., In vivo evaluation of doxorubicin-loaded polymeric micelles targeting folate receptors and early endosomal pH in drug-resistant ovarian cancer. Molecular pharmaceutics, 2009. 6(5): p. 1353-1362.

3. Bertoni, S., N. Passerini, and B. Albertini, Chapter 3 - Nanomaterials for oral drug administration, in Nanotechnology for Oral Drug Delivery, J.P. Martins and H.A. Santos, Editors. 2020, Academic Press. p. 27-76.

4. Shapira, A., et al., Beta-casein nanoparticles as an oral delivery system for chemotherapeutic drugs: impact of drug structure and properties on co-assembly. Pharmaceutical research, 2010. 27(10): p. 2175-2186.

5. Mei, L., et al., Pharmaceutical nanotechnology for oral delivery of anticancer drugs. Advanced drug delivery reviews, 2013. 65(6): p. 880-890.

6. Huang, J., et al., Layer-by-layer assembled milk protein coated magnetic nanoparticle enabled oral drug delivery with high stability in stomach and enzyme-responsive release in small intestine. Biomaterials, 2015. 39: p. 105-113.

7. Cheewatanakornkool, K., et al., Characterization and in vitro release studies of oral microbeads containing thiolated pectin-doxorubicin conjugates for colorectal cancer treatment. asian journal of pharmaceutical sciences, 2017.

12(6): p. 509-520.

8. Ferrari, M., Cancer nanotechnology: opportunities and challenges. Nature reviews cancer, 2005. 5(3): p. $161-171$.

9. Kumar, N. and R. Kumar, Chapter 4 - Nanomedicine for Cancer Treatment, in Nanotechnology and Nanomaterials in the Treatment of Life-threatening Diseases, N. Kumar and R. Kumar, Editors. 2014, William Andrew Publishing: Oxford. p. 177-246.

10. Grobmyer, S.R., B.M. Moudgil, and SpringerLink, Cancer nanotechnology : methods and protocols. 2010, New York, NY: New York, NY : Humana Press.

11. Roger, E., et al., Biopharmaceutical parameters to consider in order to alter the fate of nanocarriers after oral delivery. Nanomedicine, 2010. 5(2): p. 287-306.

12. Mei, L., et al., Pharmaceutical nanotechnology for oral delivery of anticancer drugs. Adv Drug Deliv Rev, 2013. 65(6): p. 880-90.

13. Reinholz, J., K. Landfester, and V. Mailänder, The challenges of oral drug delivery via nanocarriers. Drug delivery, 2018. 25(1): p. 1694-1705.

14. Ghosh, S., S. Ghosh, and P.C. Sil, Role of nanostructures in improvising oral medicine. Toxicology Reports, 2019. 6: p. 358-368. 
15. Xu, W., P. Ling, and T. Zhang, Polymeric Micelles, a Promising Drug Delivery System to Enhance Bioavailability of Poorly Water-Soluble Drugs. Journal of Drug Delivery, 2013. 2013: p. 340315.

16. Lee, S.C., et al., Hydrotropic polymeric micelles for enhanced paclitaxel solubility: in vitro and in vivo characterization. Biomacromolecules, 2007. 8(1): p. 202-208.

17. Zhang, Z., et al., A self-assembled nanocarrier loading teniposide improves the oral delivery and drug concentration in tumor. Journal of Controlled Release, 2013. 166(1): p. 30-37.

18. Mathot, F., et al., Intestinal uptake and biodistribution of novel polymeric micelles after oral administration. Journal of controlled release, 2006. 111(1-2): p. 47-55.

19. Mathot, F., et al., Transport mechanisms of mmePEG750P (CL-co-TMC) polymeric micelles across the intestinal barrier. Journal of controlled release, 2007. 124(3): p. 134-143.

20. Jain, A., et al., Formation and functional attributes of electrostatic complexes involving casein and anionic polysaccharides: An approach to enhance oral absorption of lycopene in rats in vivo. International journal of biological macromolecules, 2016. 93: p. 746-756.

21. Rehan, F., N. Ahemad, and M. Gupta, Casein Nanomicelle as an emerging biomaterial-A comprehensive review. Colloids and Surfaces B: Biointerfaces, 2019.

22. Penalva, R., et al., Casein nanoparticles as carriers for the oral delivery of folic acid. Food Hydrocolloids, 2015. 44(Supplement C): p. 399-406.

23. Głąb, T.K. and J. Boratyński, Potential of Casein as a Carrier for Biologically Active Agents. Topics in Current Chemistry, 2017. 375(4): p. 71.

24. Liu, C., et al., Self-Assembled Casein Nanoparticles Loading Triptolide for the Enhancement of Oral Bioavailability. Natural Product Communications, 2020. 15(8): p. 1934578X20948352.

25. Malekhosseini, P., et al., Development of casein-based nanoencapsulation systems for delivery of epigallocatechin gallate and folic acid. Food science \& nutrition, 2019. 7(2): p. 519-527.

26. Peñalva, R., et al., Increased oral bioavailability of resveratrol by its encapsulation in casein nanoparticles. International journal of molecular sciences, 2018. 19(9): p. 2816.

27. Semo, E., et al., Casein micelle as a natural nano-capsular vehicle for nutraceuticals. Food Hydrocolloids, 2007. 21(5): p. 936-942.

28. Shapira, A., et al., $\beta$-Casein nanoparticle-based oral drug delivery system for potential treatment of gastric carcinoma: Stability, target-activated release and cytotoxicity. European Journal of Pharmaceutics and Biopharmaceutics, 2012. 80(2): p. 298-305.

29. Esmaili, M., et al., Beta casein-micelle as a nano vehicle for solubility enhancement of curcumin; food industry application. LWT-food science and technology, 2011. 44(10): p. 2166-2172.

30. Pan, K., Q. Zhong, and S.J. Baek, Enhanced dispersibility and bioactivity of curcumin by encapsulation in casein nanocapsules. Journal of agricultural and food chemistry, 2013. 61(25): p. 6036-6043. 
31. Chen, H., Y. Zhang, and Q. Zhong, Physical and antimicrobial properties of spray-dried zein-casein nanocapsules with co-encapsulated eugenol and thymol. Journal of Food Engineering, 2015. 144(Supplement C): p. 93-102.

32. Chen, L., et al., Casein nanoparticles as oral delivery carriers of mequindox for the improved bioavailability. Colloids and Surfaces B: Biointerfaces, 2020. 195: p. 111221.

33. Elbialy, N.S. and N. Mohamed, Alginate-coated caseinate nanoparticles for doxorubicin delivery: Preparation, characterisation, and in vivo assessment. International Journal of Biological Macromolecules, 2020.

34. Hira, S.K., et al., Targeted delivery of doxorubicin-loaded poly ( $\varepsilon$-caprolactone)-b-poly (N-vinylpyrrolidone) micelles enhances antitumor effect in lymphoma. Plos one, 2014. 9(4): p. e94309.

35. Zhang, J., et al., Cisplatin and doxorubicin high-loaded nanodrug based on biocompatible thioether-and ethanebridged hollow mesoporous organosilica nanoparticles. Journal of colloid and interface science, 2018. 513: p. 214221.

36. Rehan, F., et al., Optimization and Formulation of Nanostructured and Self-Assembled Caseinate Micelles for Enhanced Cytotoxic Effects of Paclitaxel on Breast Cancer Cells. Pharmaceutics, 2020. 12(10).

37. Scheeren, L.E., et al., Comparative study of reversed-phase high-performance liquid chromatography and ultraviolet-visible spectrophotometry to determine doxorubicin in pH-sensitive nanoparticles. Analytical Letters, 2018. 51(10): p. 1445-1463.

38. Elzoghby, A.O., W.M. Samy, and N.A. Elgindy, Novel spray-dried genipin-crosslinked casein nanoparticles for prolonged release of alfuzosin hydrochloride. Pharmaceutical Research, 2013. 30(2): p. 512-522.

39. Rehan, F., et al., Optimization and formulation of nanostructured and self-assembled caseinate micelles for enhanced cytotoxic effects of paclitaxel on breast cancer cells. Pharmaceutics, 2020. 12(10): p. 984.

40. Quintás, G., et al., Fourier transform infrared determination of imidacloprid in pesticide formulations. Journal of the Brazilian Chemical Society, 2004. 15(2): p. 307-312.

41. Fatemian, T. and E.H. Chowdhury, Cytotoxicity Enhancement in Breast Cancer Cells with Carbonate ApatiteFacilitated Intracellular Delivery of Anti-Cancer Drugs. Toxics, 2018. 6(1).

42. El-Far, S.W., et al., Phytosomal bilayer-enveloped casein micelles for codelivery of monascus yellow pigments and resveratrol to breast cancer. Nanomedicine (Lond), 2018. 13(5): p. 481-499.

43. Cui, C., et al., Cellular uptake, intracellular trafficking, and antitumor efficacy of doxorubicin-loaded reductionsensitive micelles. Biomaterials, 2013. 34(15): p. 3858-3869.

44. Bae, Y., et al., Design of environment-sensitive supramolecular assemblies for intracellular drug delivery: Polymeric micelles that are responsive to intracellular pH change. Angewandte Chemie International Edition, 2003. 42(38): p. 4640-4643.

45. Oecd, OECD Guidelines for the Testing of Chemicals. 1994: Organization for Economic.

46. Roux, S., E. Sablé, and R.D. Porsolt, Primary observation (Irwin) test in rodents for assessing acute toxicity of a test agent and its effects on behavior and physiological function. Current Protocols in Pharmacology, 2004. 27(1): p. 10.10. 1-10.10. 23. 
47. Anadón, A., et al., Acute Oral Safety Study of Sodium Caseinate Glycosylated via Maillard Reaction with Galactose in Rats. Journal of food protection, 2014. 77(3): p. 472-479.

48. Casanova, F., Colloidal stability of native and cross-linked casein micelles and their potential use as nanocarrier for cyanidin-3-0 glucoside. 2017, Universidade Federal de Viçosa.

49. Casanova, F., et al., pH-and ionic strength-dependent interaction between cyanidin-3-O-glucoside and sodium caseinate. Food chemistry, 2018. 267: p. 52-59.

50. Wang, J., et al., A micelle self-assembled from doxorubicin-arabinoxylan conjugates with ph-cleavable bond for synergistic antitumor therapy. Nanoscale Research Letters, 2017. 12(1): p. 1-9.

51. Ali, O.M., et al., Synthesis of Lactoferrin Mesoporous Silica Nanoparticles for Pemetrexed/Ellagic Acid Synergistic Breast Cancer Therapy. Colloids and Surfaces B: Biointerfaces, 2020: p. 110824.

52. Shi, P. and J.C. Goh, Release and cellular acceptance of multiple drugs loaded silk fibroin particles. International journal of pharmaceutics, 2011. 420(2): p. 282-289.

53. Jain, S., et al., pH dependent drug release from drug conjugated PEGylated CdSe/ZnS nanoparticles. Materials Chemistry and Physics, 2020. 240: p. 122162.

54. Victor, S.P., et al., Supramolecular hydroxyapatite complexes as theranostic near-infrared luminescent drug carriers. CrystEngComm, 2014. 16(38): p. 9033-9042.

55. Gnapareddy, B., et al., Chemical and physical characteristics of doxorubicin hydrochloride drug-doped salmon DNA thin films. Scientific reports, 2015. 5: p. 12722.

56. Gandhi, S. and I. Roy, Doxorubicin-loaded casein nanoparticles for drug delivery: Preparation, characterization and in vitro evaluation. International journal of biological macromolecules, 2019. 121: p. 6-12.

57. Elzoghby, A.O., et al., Novel ionically crosslinked casein nanoparticles for flutamide delivery: formulation, characterization, and in vivo pharmacokinetics. International journal of nanomedicine, 2013. 8: p. 1721.

58. Raj, J. and K.B. Uppuluri, Metformin loaded casein micelles for sustained delivery: formulation, characterization and in-vitro evaluation. Biomedical and Pharmacology Journal, 2015. 8(1): p. 83-89.

59. Abdelmoneem, M.A., et al., Dual-targeted casein micelles as green nanomedicine for synergistic phytotherapy of hepatocellular carcinoma. Journal of Controlled Release, 2018. 287: p. 78-93.

60. Liu, J., et al., Combining spatially resolved hydrochemical data with in-vitro nanoparticle stability testing: Assessing environmental behavior of functionalized gold nanoparticles on a continental scale. Environment International, 2013. 59: p. 53-62.

61. Hossain, S., et al., Fabrication and intracellular delivery of doxorubicin/carbonate apatite nanocomposites: effect on growth retardation of established colon tumor. PLoS One, 2013. 8(4): p. e60428.

62. Tang, L., et al., Targeting tumor vasculature with aptamer-functionalized doxorubicin-polylactide nanoconjugates for enhanced cancer therapy. ACS nano, 2015. 9(5): p. 5072-5081. 
63. Chaudhary, A., et al., One pot synthesis of doxorubicin loaded gold nanoparticles for sustained drug release. RSC Advances, 2015. 5(118): p. 97330-97334.

64. Kataoka, K., et al., Doxorubicin-loaded poly (ethylene glycol)-poly ( $\beta$-benzyl-I-aspartate) copolymer micelles: their pharmaceutical characteristics and biological significance. Journal of Controlled Release, 2000. 64(1-3): p. $143-153$.

65. Amjad, M.W., et al., Doxorubicin-loaded cholic acid-polyethyleneimine micelles for targeted delivery of antitumor drugs: synthesis, characterization, and evaluation of their in vitro cytotoxicity. Nanoscale Research Letters, 2012. 7(1): p. 687.

66. Sun, C.Z., et al., Characterization of the doxorubicin-pluronic F68 conjugate micelles and their effect on doxorubicin resistant human erythroleukemic cancer cells. Journal of Nanomedicine and Nanotechnology, 2011. 2: p. 1000114.

67. Bakhtiar, A. and E.H. Chowdhury, PH-responsive strontium nanoparticles for targeted gene therapy against mammary carcinoma cells. Asian journal of pharmaceutical sciences, 2021. 16(2): p. 236-252.

68. El-Far, S.W., et al., Phytosomal bilayer-enveloped casein micelles for codelivery of monascus yellow pigments and resveratrol to breast cancer. Nanomedicine, 2018. 13(5): p. 481-499.

69. Mohapatra, S., et al., Doxorubicin loaded carboxymethyl Assam bora rice starch coated superparamagnetic iron oxide nanoparticles as potential antitumor cargo. Heliyon, 2019. 5(6): p. e01955.

70. Picchio, M.L., et al., Crosslinked casein-based micelles as a dually responsive drug delivery system. Polymer Chemistry, 2018. 9(25): p. 3499-3510.

71. Komuro, H., et al., The effect of glucose modification of hydroxyapatite nanoparticles on gene delivery. Journal of Biomedical Materials Research Part A, 2019. 107(1): p. 61-66.

72. Chowdhury, E.H., Nanotherapeutics: from laboratory to clinic. 2016: CRC press.

73. Swarnakar, N.K., K. Thanki, and S. Jain, Bicontinuous cubic liquid crystalline nanoparticles for oral delivery of doxorubicin: implications on bioavailability, therapeutic efficacy, and cardiotoxicity. Pharmaceutical research, 2014. 31(5): p. 1219-1238.

74. Kanwal, U., et al., Advances in nano-delivery systems for doxorubicin: an updated insight. Journal of Drug Targeting, 2018. 26(4): p. 296-310.

75. Souris, J.S., et al., Surface charge-mediated rapid hepatobiliary excretion of mesoporous silica nanoparticles. Biomaterials, 2010. 31(21): p. 5564-5574.

76. Ambruosi, A., H. Yamamoto, and J. Kreuter, Body distribution of polysorbate-80 and doxorubicin-loaded [14C] poly (butyl cyanoacrylate) nanoparticles after iv administration in rats. Journal of drug targeting, 2005. 13(10): p. 535-542.

77. Perlstein, H., et al., Beta-casein nanocarriers of celecoxib for improved oral bioavailability. European Journal of Nanomedicine, 2014. 6(4): p. 217-226.

78. Bar-Zeev, M., Y.G. Assaraf, and Y.D. Livney, $\beta$-casein nanovehicles for oral delivery of chemotherapeutic drug combinations overcoming P-glycoprotein-mediated multidrug resistance in human gastric cancer cells. Oncotarget, 2016. 7(17): p. 23322. 
79. Lee, M.-Y., et al., A 4-week repeated dose oral toxicity and cytotoxicity study of gumiganghwaltang in Crl: CD (SD) rats. Toxicology international, 2011. 18(2): p. 146.

80. Kim, J.-C., et al., Subchronic toxicity of plant sterol esters administered by gavage to Sprague-Dawley rats. Food and chemical toxicology, 2002. 40(11): p. 1569-1580.

\section{Figures}

A
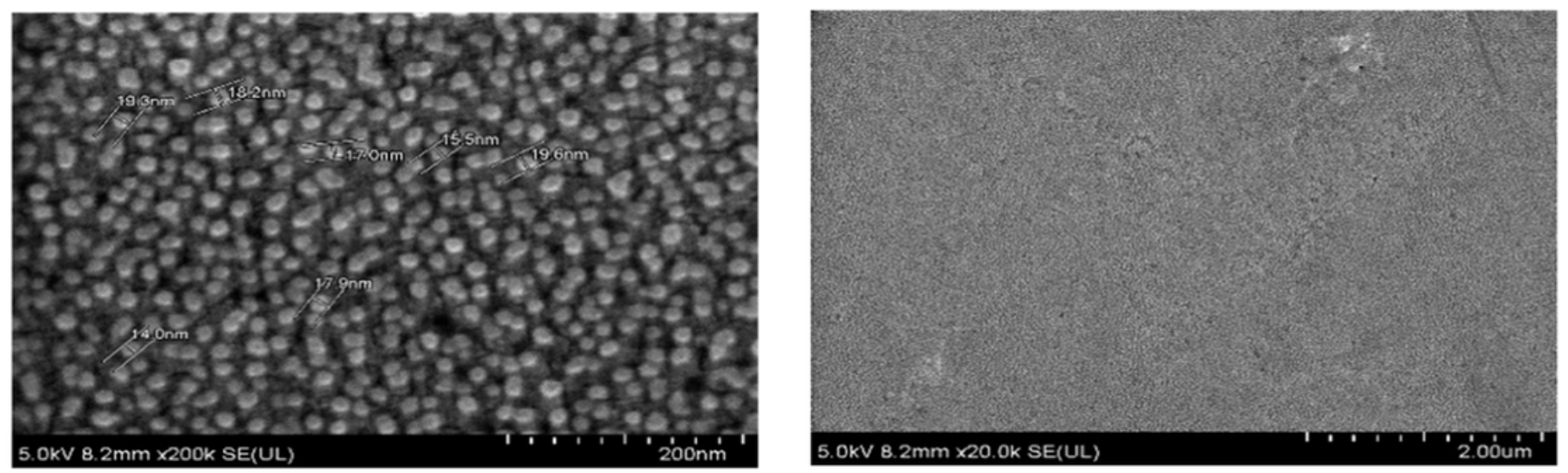

B
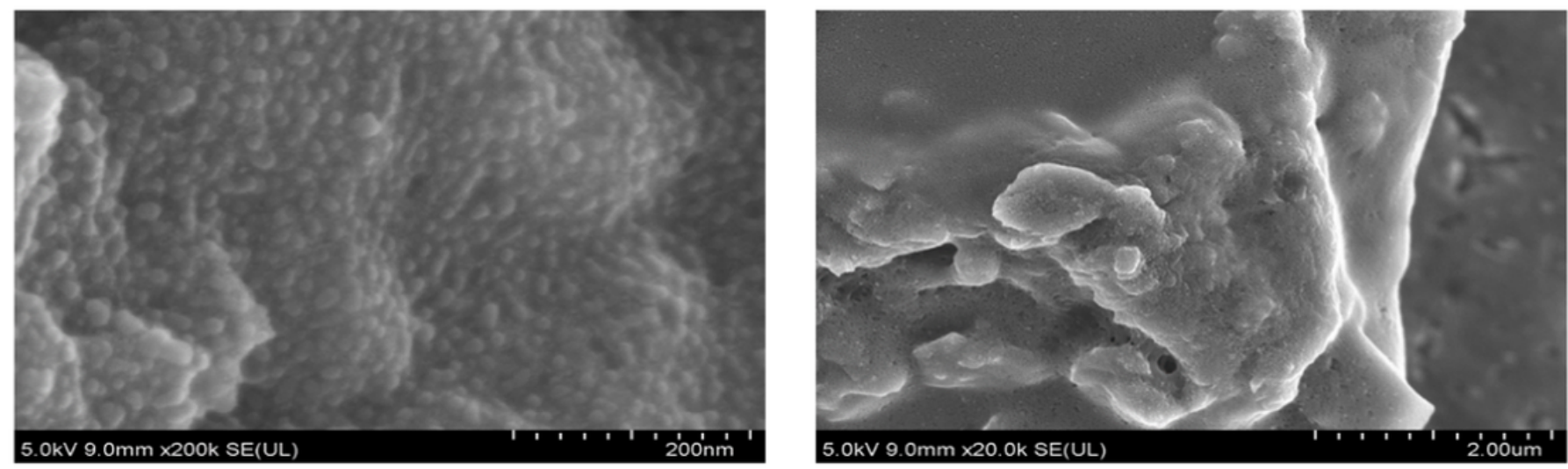

C
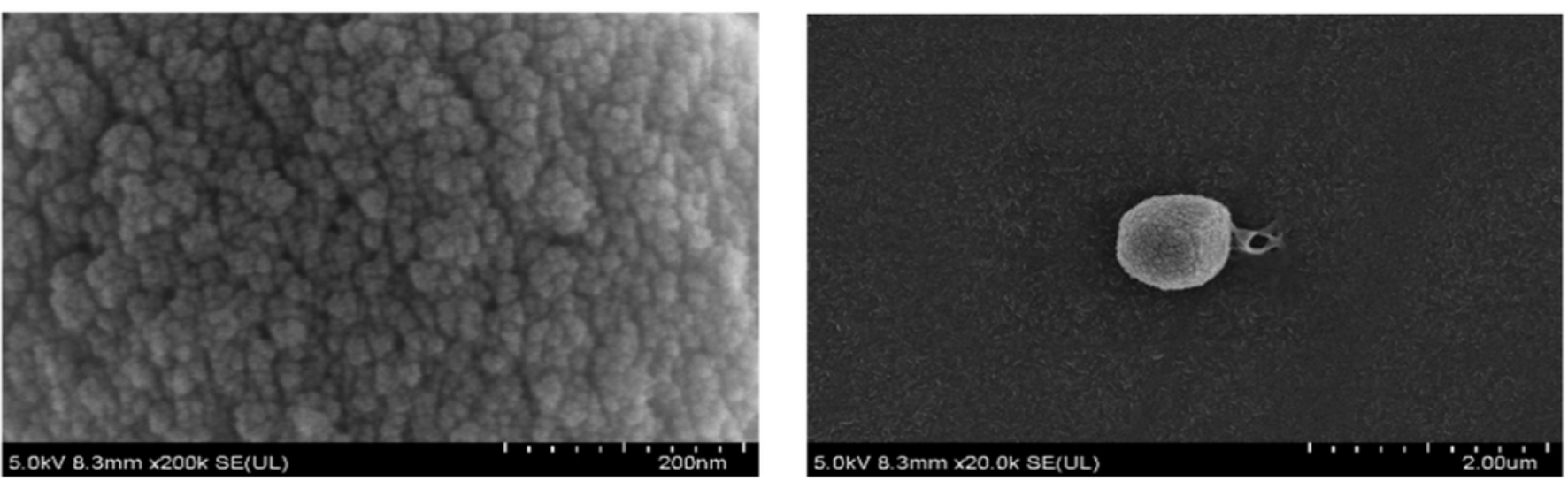

Figure 1

FESEM micrographs of (A) DOX-NaCNs (B) Blank NaCNs (C) Negative Control (DOX+ water). Scale bar: $200 \mathrm{~nm}$ and 2 $\mu \mathrm{m}$. The samples $(10 \mu \mathrm{l})$ were placed on glass covers and air-dried at an ambient temperature for FESEM analysis. 


\section{A}
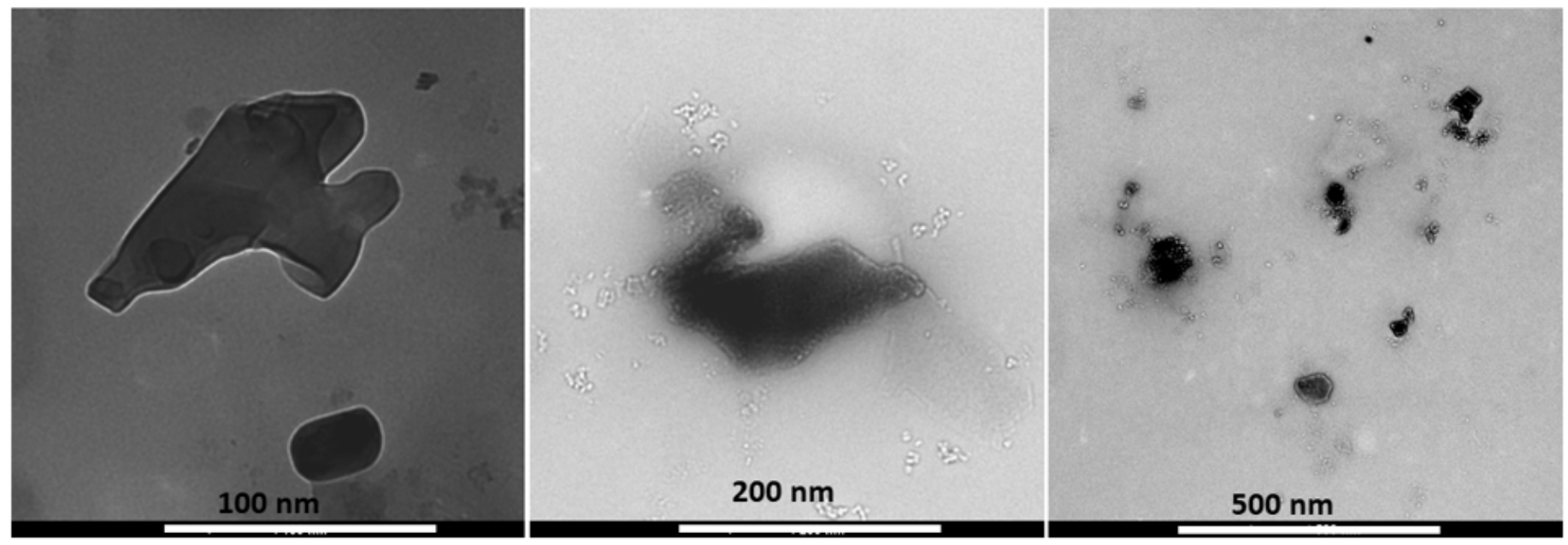

B
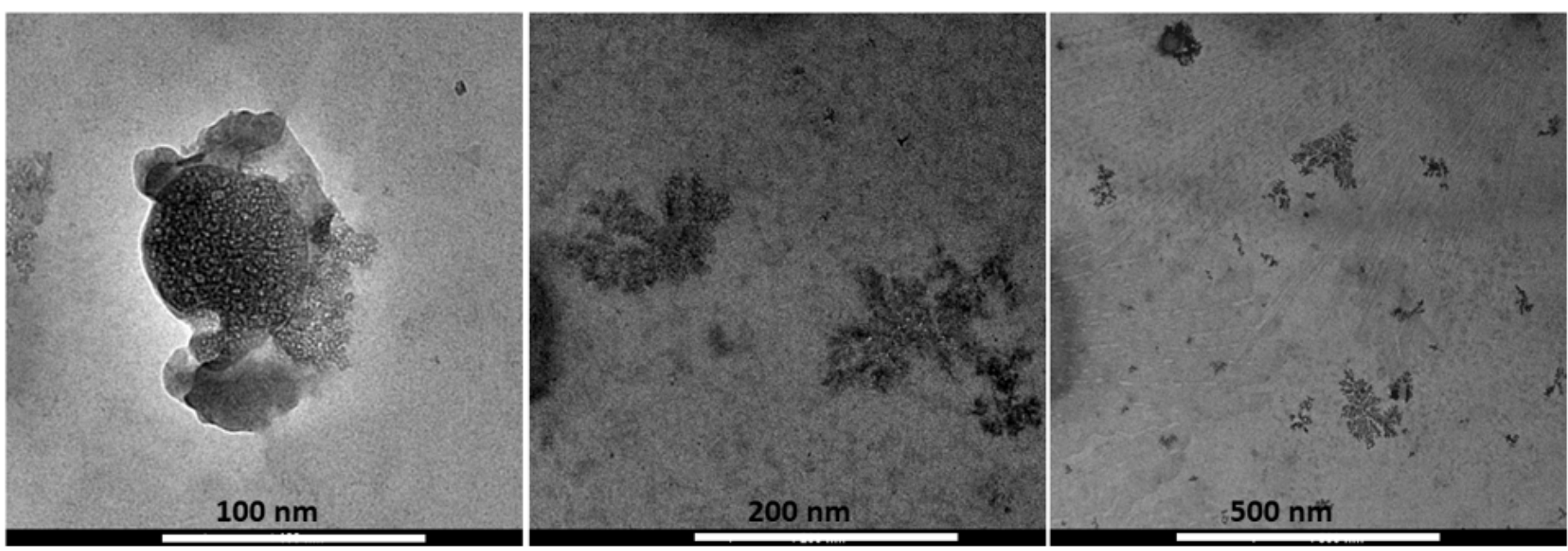

\section{Figure 2}

HR-TEM micrograph of (A) blank NaCNs (B) DOX-NaCNs showing spherical shaped nanomicelles. Scale bars: 100, 200 and $500 \mathrm{~nm}$. For analysis purposes, copper grid was suspended in each sample for 30 secs, were left at room temperature for an overnight air drying before HR-TEM analysis. 


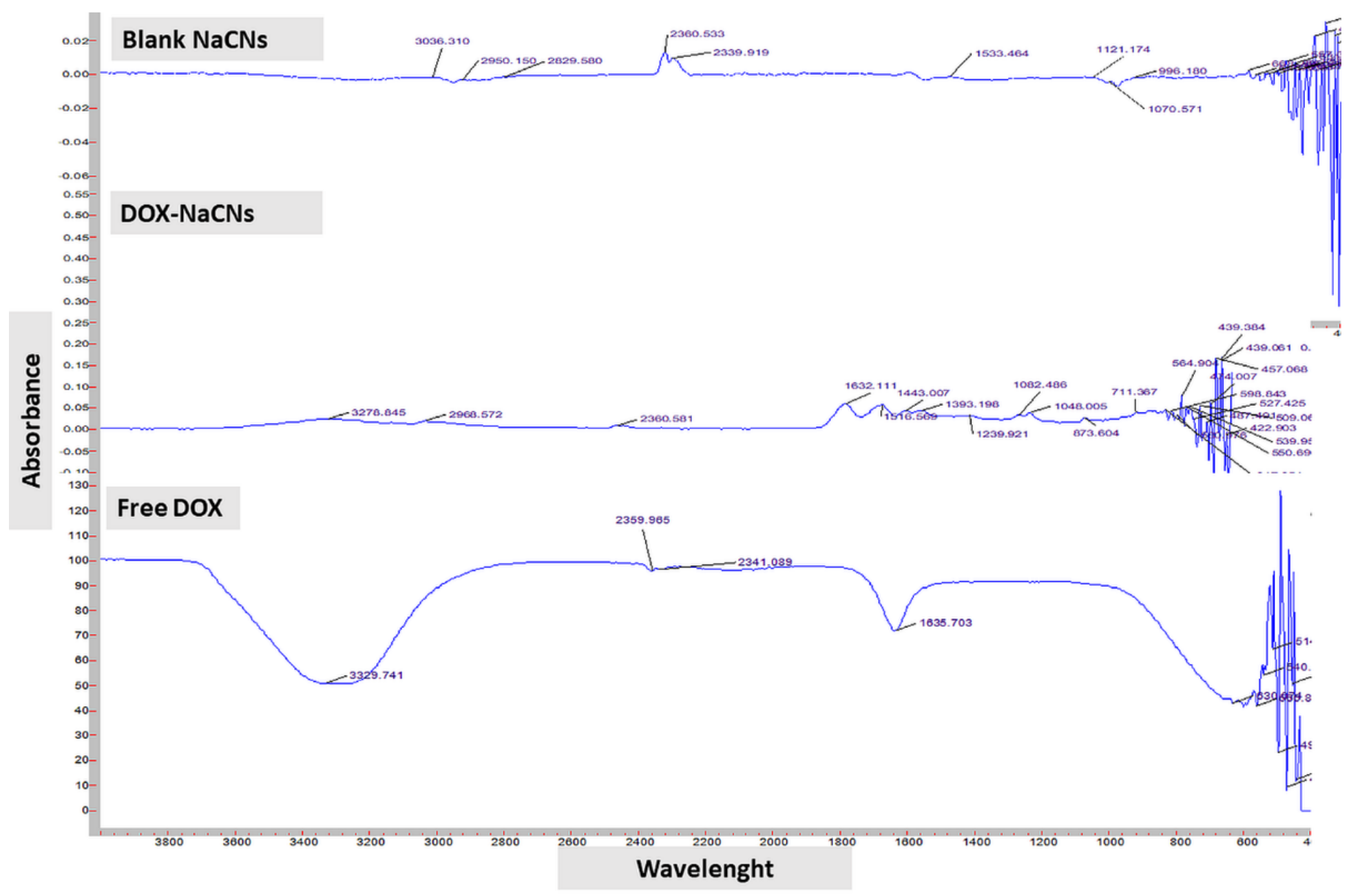

Figure 3

FTIR spectra of blank NaCNs, DOX-NaCNs and Free DOX. For FT-IR analysis, the samples were prepared and freezedried for two days. Subsequently, the lyophilized powder were analyzed using an FT-IR machine. The spectrum was recorded (Varian Resolution Pro 640 software, version 5.1, Agilent, Santa Clara, CA, USA) over the range of 500$4000 / \mathrm{cm}$. 
A

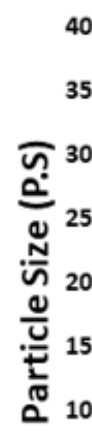

50

0
B

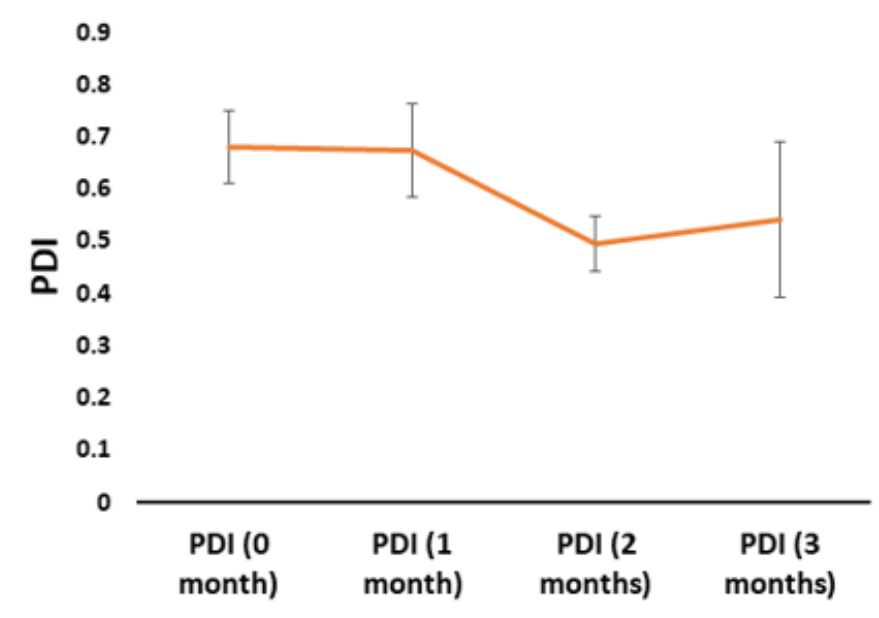

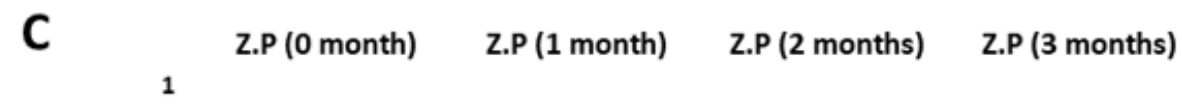

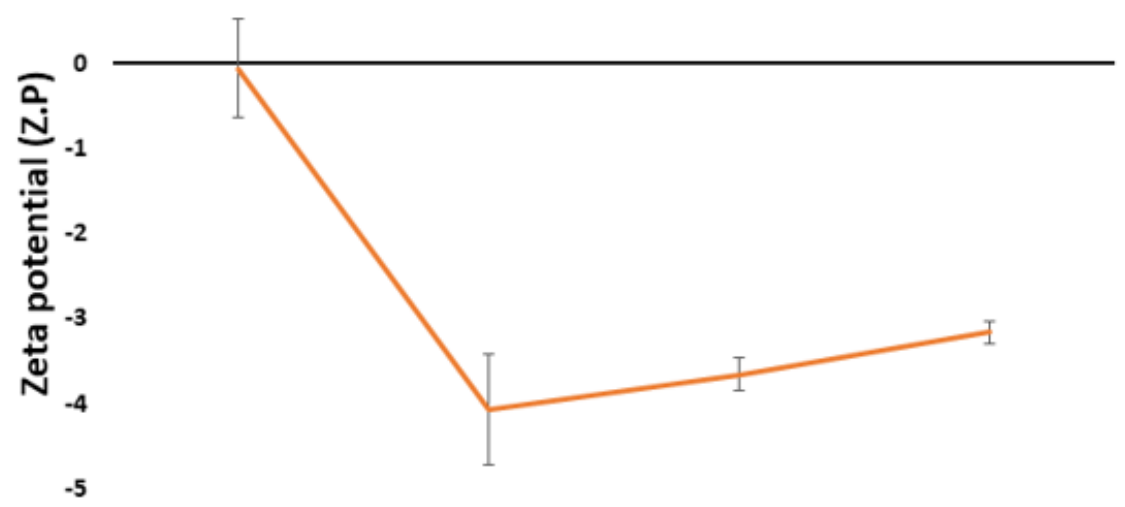

Figure 4

Stability studies of the DOX-NaCNs at regular intervals of one month up to 3 months, where P.S, PDI and Z.P denote particle size, polydispersity index and zeta potential respectively. The samples were diluted with water (1:10) at ambient temperature and then measured by zetasizer. 
A

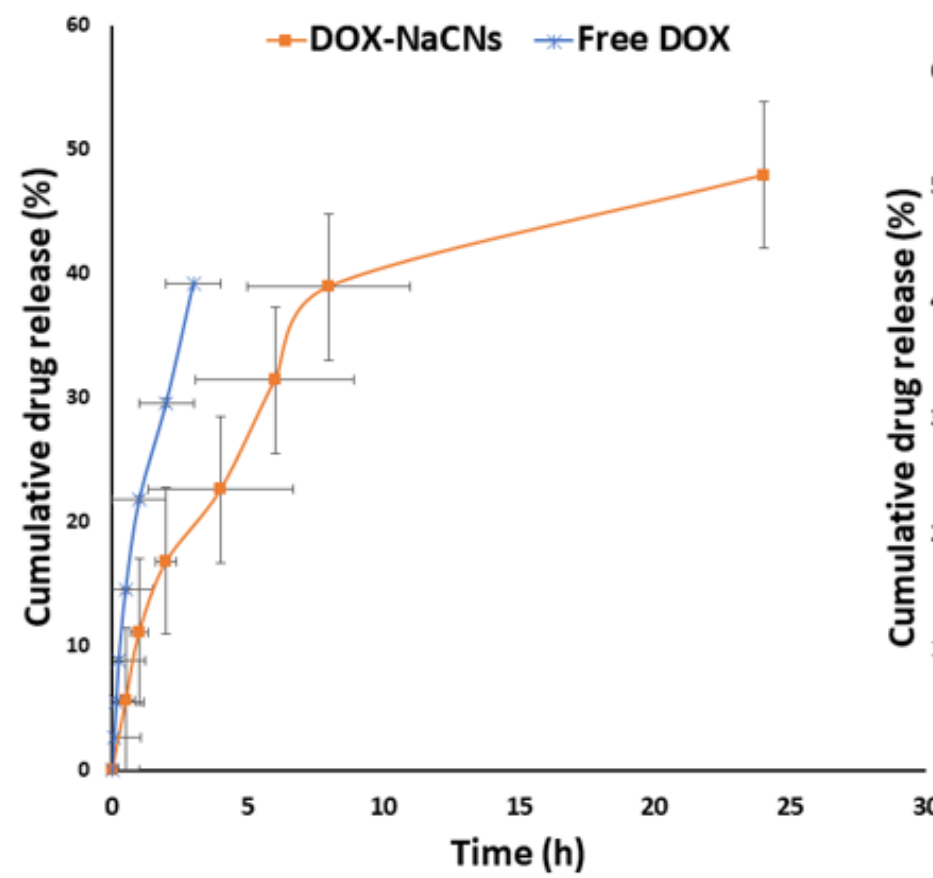

B

- -DOX-NaCNs * Free DOX

60

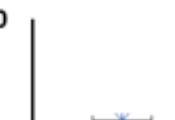

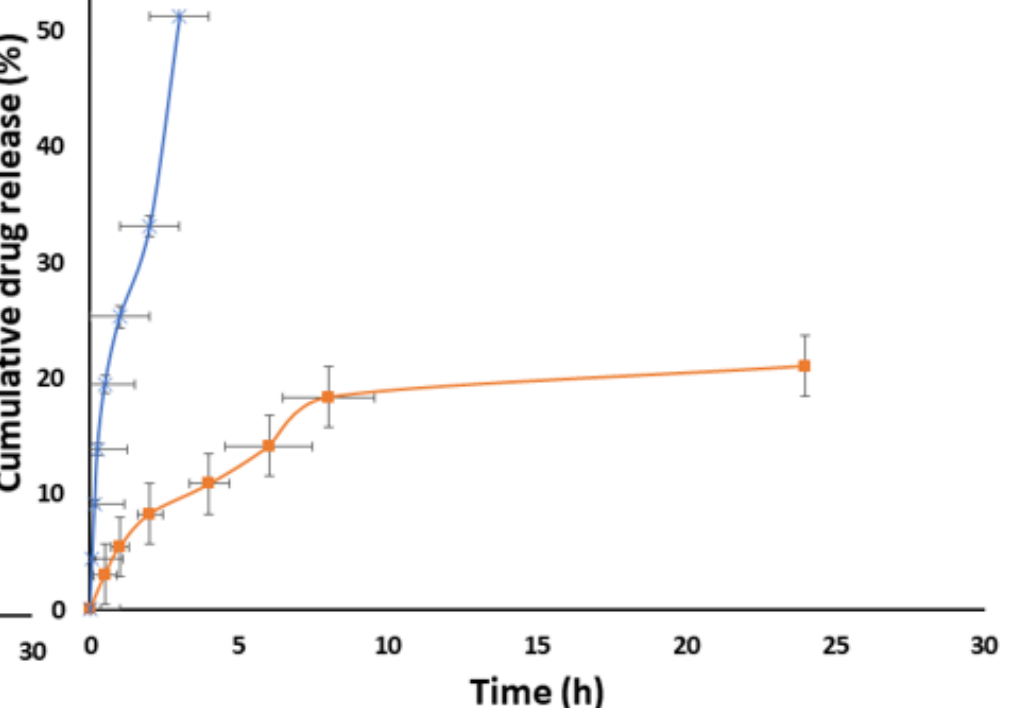

\section{Figure 5}

In-vitro release profile of $\mathrm{DOX}-\mathrm{NaCNs}$ at $\mathrm{pH}$ (A) 7.4 and (B) 5.0. Samples were prepared and sealed inside the dialysis membrane. Later, the dialysis bag was tied to the paddle of USP XXIV dissolution apparatus II and dialyzed against $250 \mathrm{~mL}$ of phosphate-buffered saline $\left(\mathrm{pH} \mathrm{7.4/5.0)}\right.$ at $37 \pm 2^{\circ} \mathrm{C}$ and under continuous magnetic stirring (100 rpm) for 24 hours. 
A

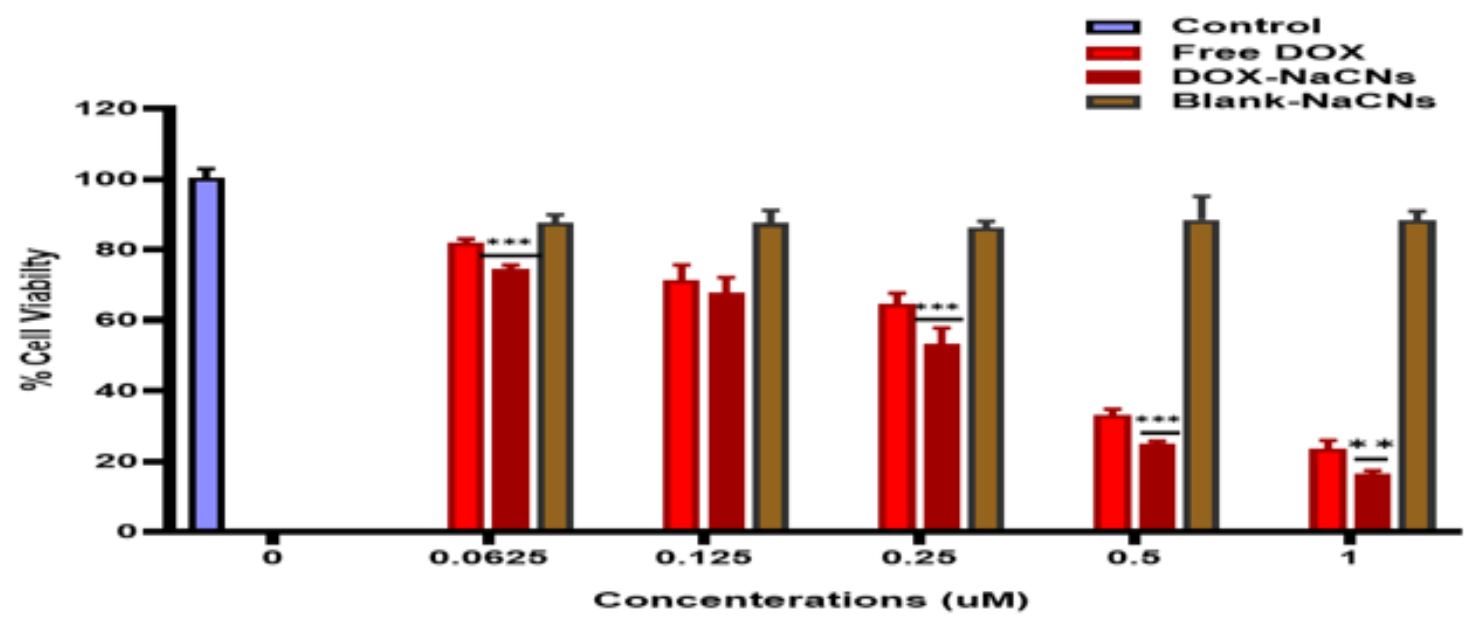

B

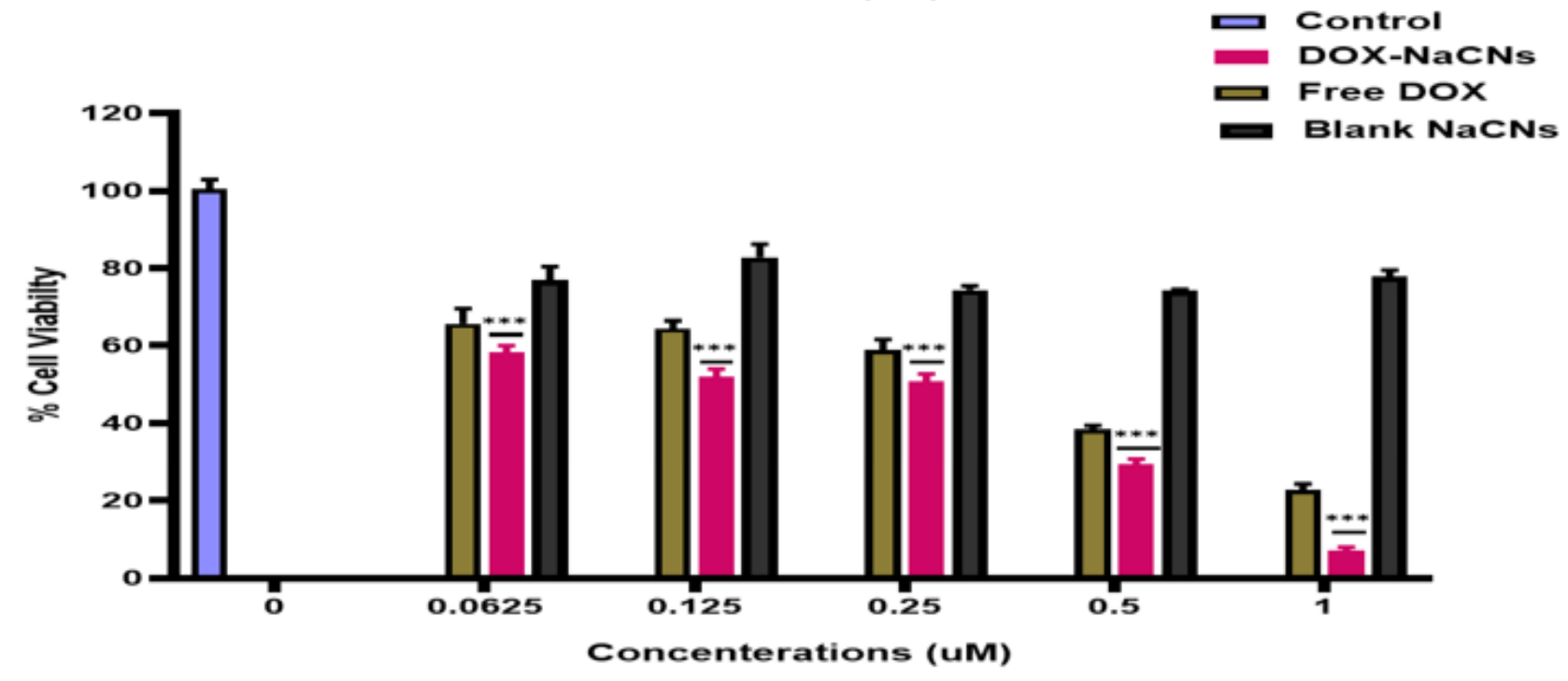

Figure 6

In vitro cell viability analysis through an MTT assay of blank NaCNs, free DOX and DOX- NaCNs on (A) MCF-7 and (B) MDA-MB 231 cell lines $0.0625-1.0000 \mu \mathrm{M}$ after $48 \mathrm{~h}$ incubation. Data were shown as mean \pm SD where $n \geq 3$ and ( $* \star \star)$ is $p<0.001$ and $(* *)$ is $p<0.01$ vs free DOX. 
$4 \mathrm{~h}$

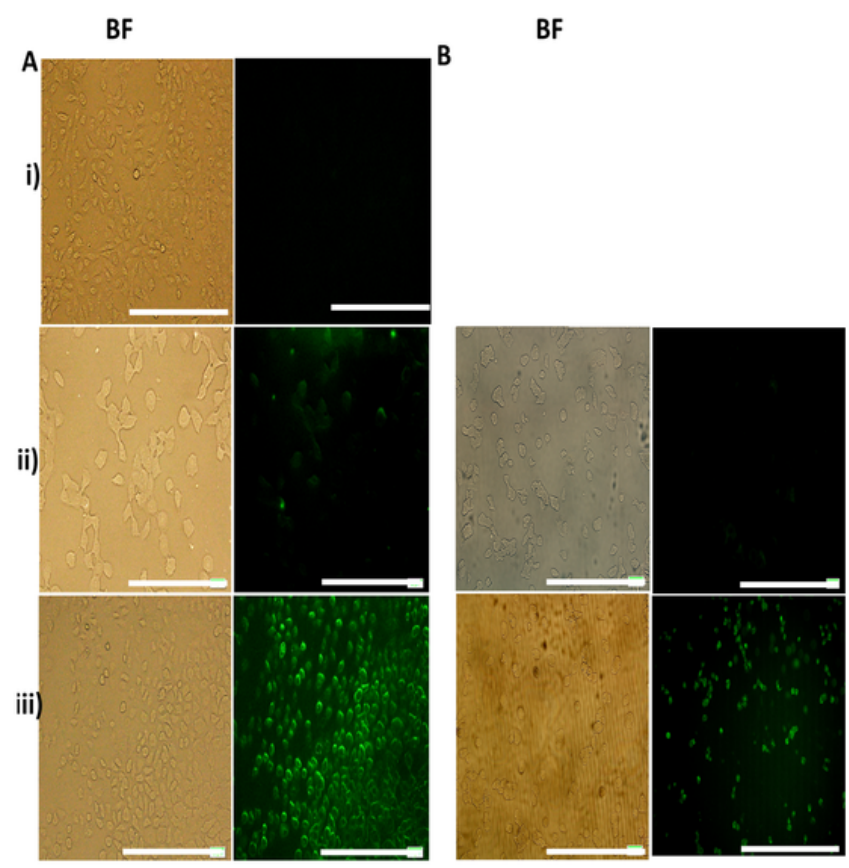

$24 \mathrm{~h}$

A BF

$\mathrm{BF}$

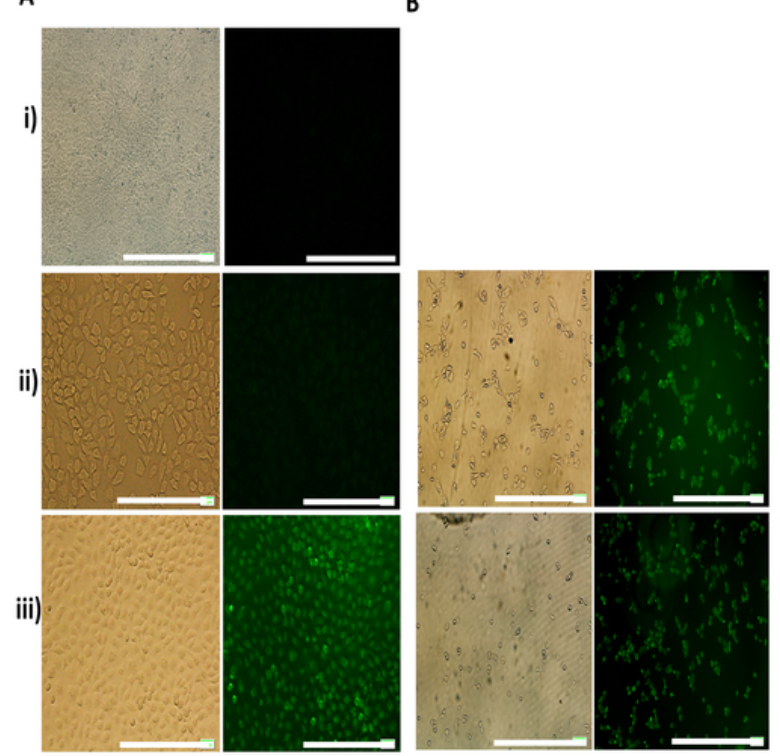

Figure 7

Fluorescence images of MCF-7 cells treated with i) media only (control) ii) free DOX iii) DOX-NaCNs at (A) $5 \mu \mathrm{m}$ and (B) $10 \mu \mathrm{M}$; observed after $4 \mathrm{~h}$ and $24 \mathrm{~h}$ of treatment with $10 \mathrm{x}$ magnification at a scale bar of $50 \mu \mathrm{m}$. 

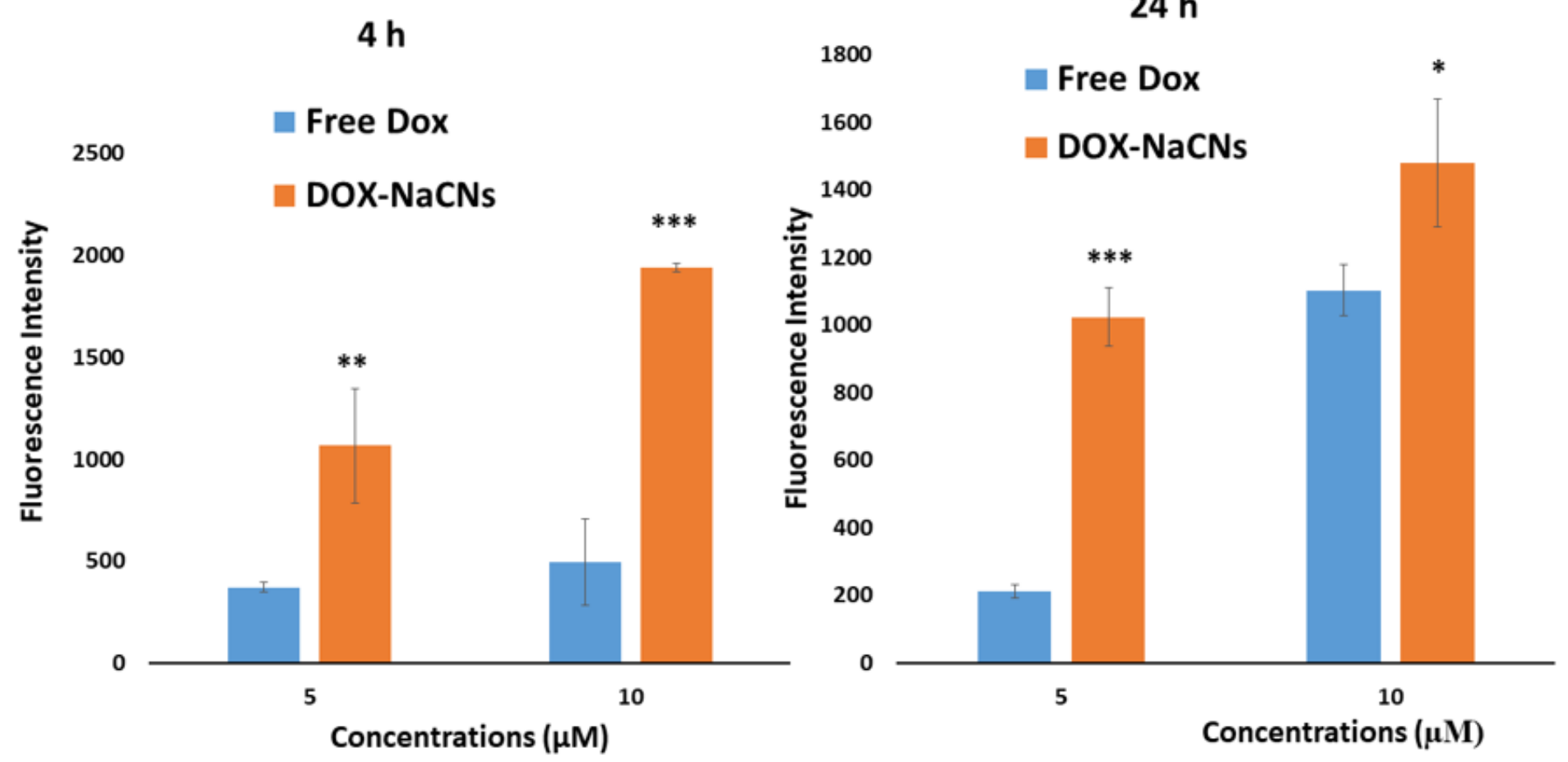

Figure 8

Cellular uptake of free DOX and DOX-NaCNs at 5 and $10 \mu \mathrm{M}$ observed after 4 and $24 \mathrm{~h}$ of treatment. Data were shown as mean \pm SD where $n=3$ and $(* \star *)$ is $p<0.001$ and $(* *)$ is $p<0.01$ vs free DOX.

(A)

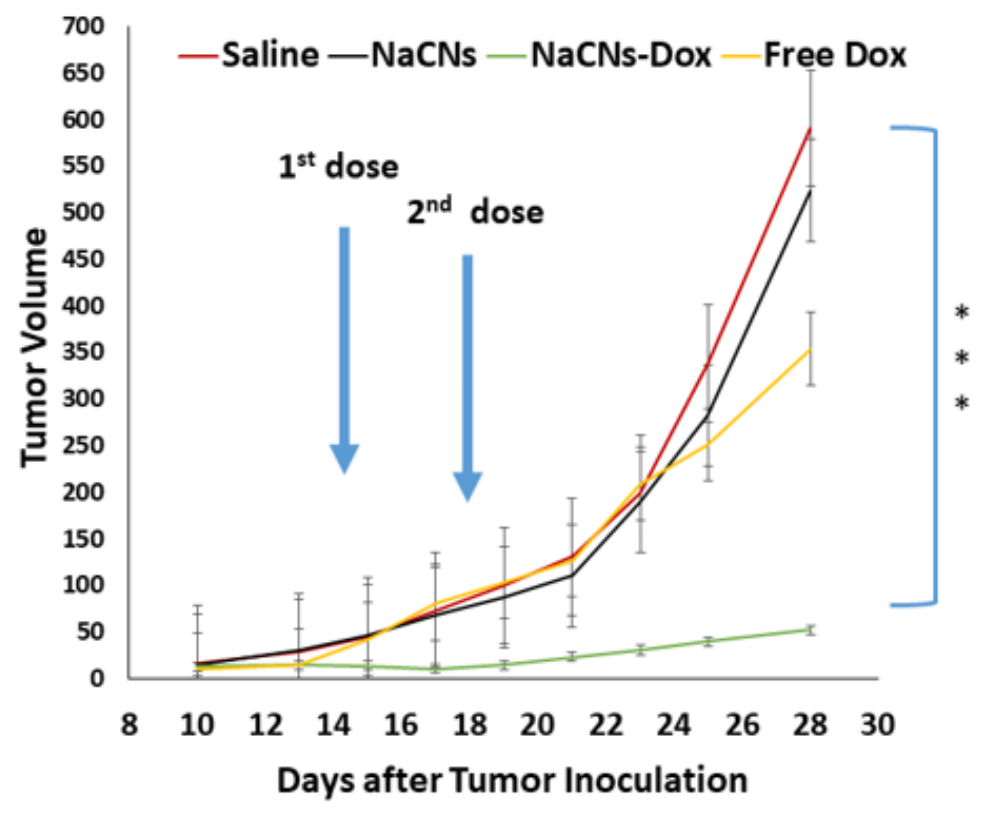

(B)

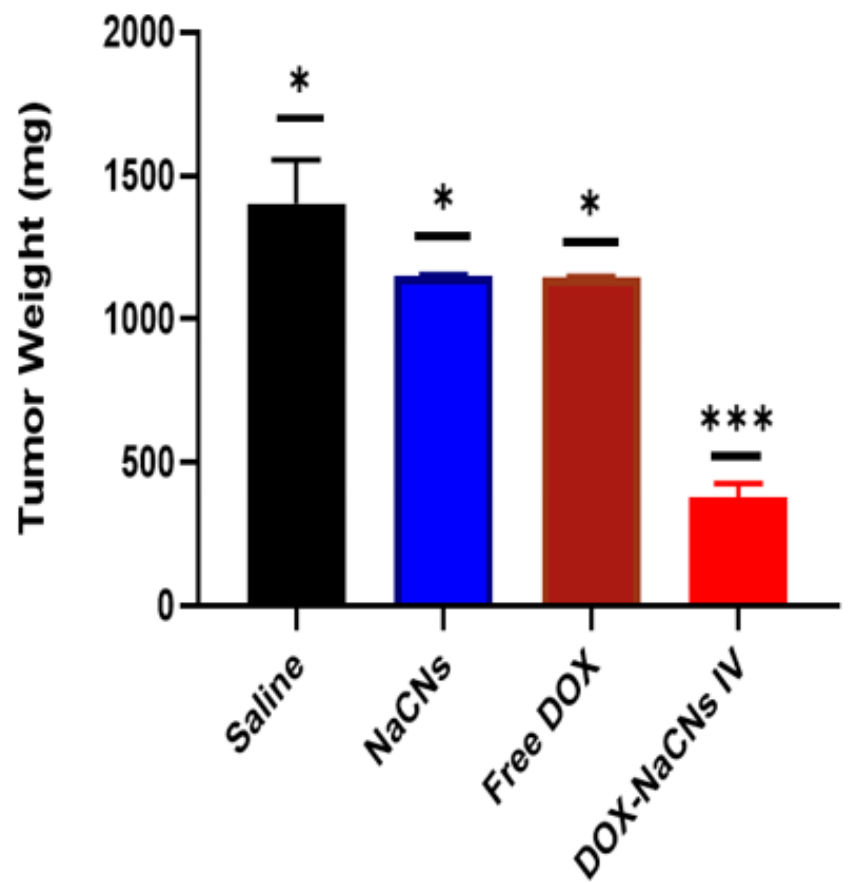

Figure 9 
In vivo tumour growth inhibition of free DOX and DOX-NaCNs following IV treatment : In vivo anti-tumour effects showing $(A)$ mean tumour volume $(\mathrm{mm} 3)$ of negative control, NaCNS, free DOX and DOX-NaCNs-treated groups throughout the experimental period following intravenous administration and $(B)$ the mean weight of the tumour of negative control, NaCNs, free DOX and DOX-NaCNs used to treat the mice on day 28 after the mice were sacrificed [values were considered statistically significant $(*)$ at $p<0.05$, very significant $(* *)$ at $p<0.01$ and highly significant $(* \star *)$ at $p<0.001]$.

\section{Saline}

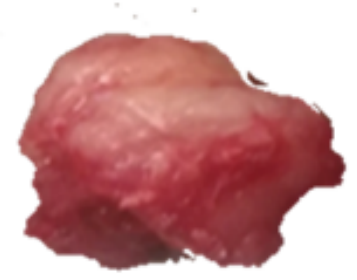

$\mathrm{NaCNs}$

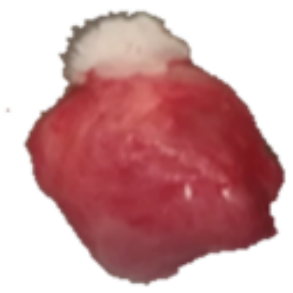

Free DOX

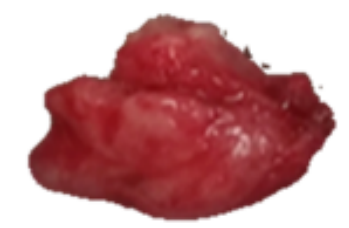

\section{DOX-}

NaCNs IV

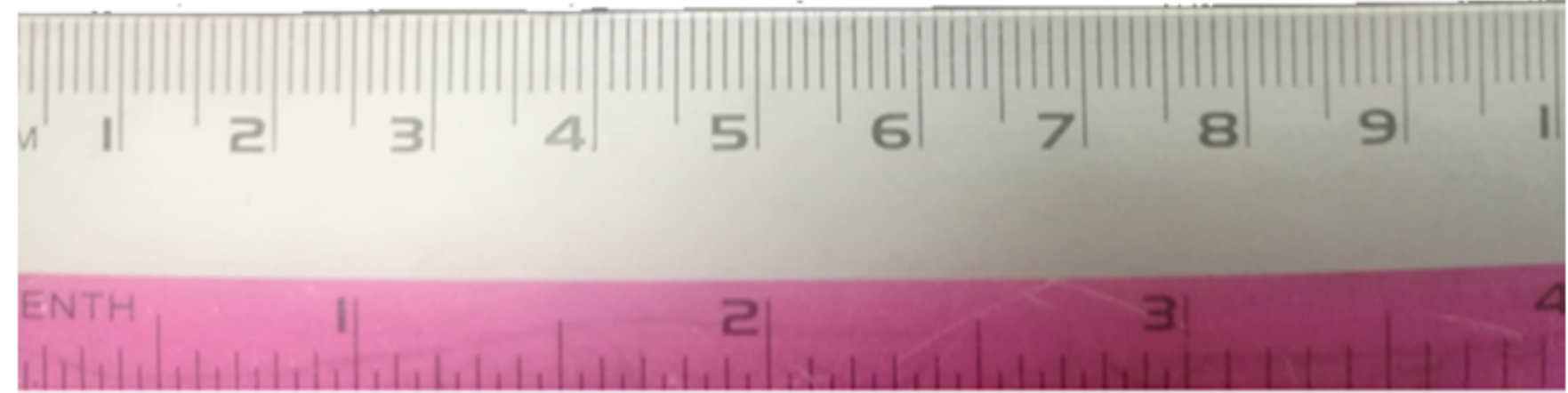

\section{Figure 10}

Visual images of excised tumour illustrating the different anti-tumour effects between the groups treated intravenously with DOX-NaCNs when compared with the free DOX, NaCNs and saline orally treated groups over the 28 days treatment period. 
(A)

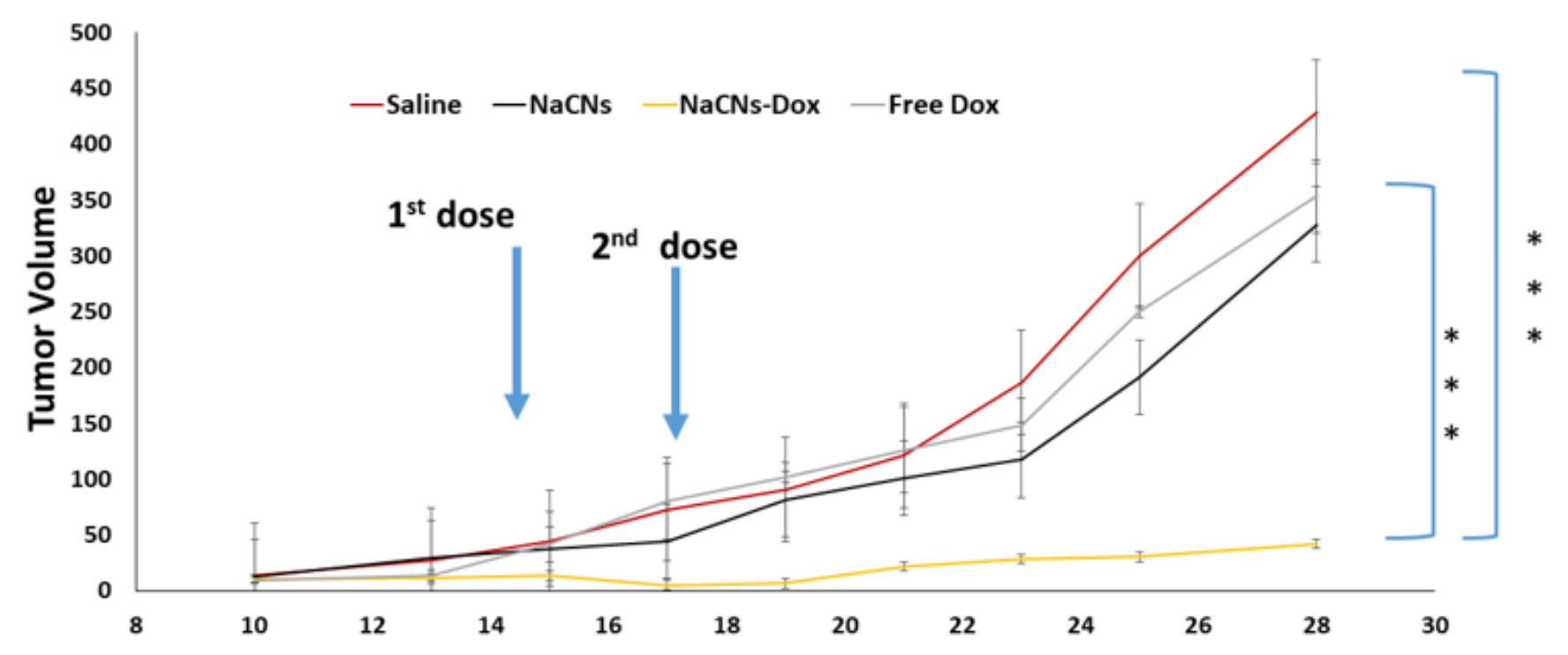

(B)

Days after Tumor Inoculation

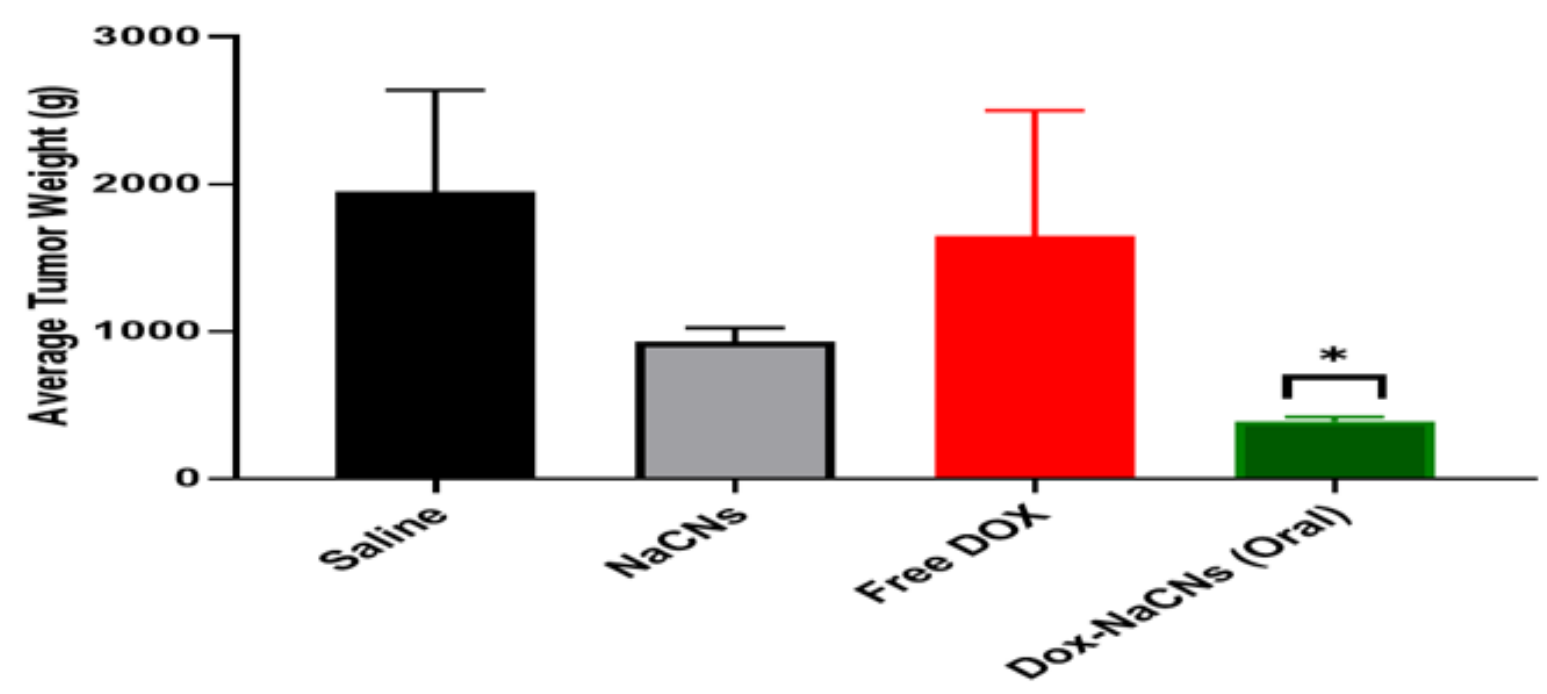

Figure 11

In vivo tumour growth inhibition of free DOX and DOX-NaCNs following oral treatment : In vivo anti-tumour effects (A) average tumour volume (mm3) for negative control compared with NaCNS, free DOX and DOX-NaCNs given orally B) the mean weight of the tumour of negative control vs NaCNs, free DOX and DOX-NaCNs. Data were presented as mean $\pm S D$ and were considered as statistically significant $(*)$ at $p<0.05$, very significant $(* *)$ at $p<0.01$ and highly significant $(\star \star \star)$ at $p<0.001$. 


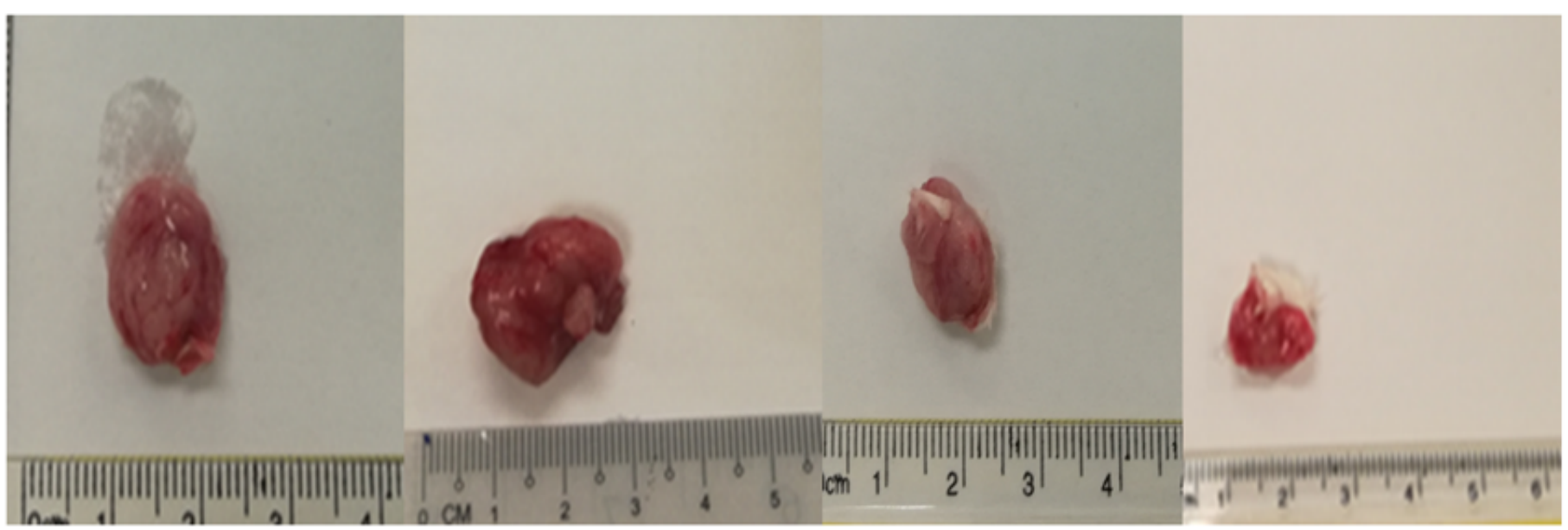

Figure 12

Visual images of excised tumour images illustrating the different anti-tumour effects between the groups orally treated with DOX-NaCNs when compared with the free DOX, NaCNs and saline-treated groups over the 28 days treatment period.

(A)
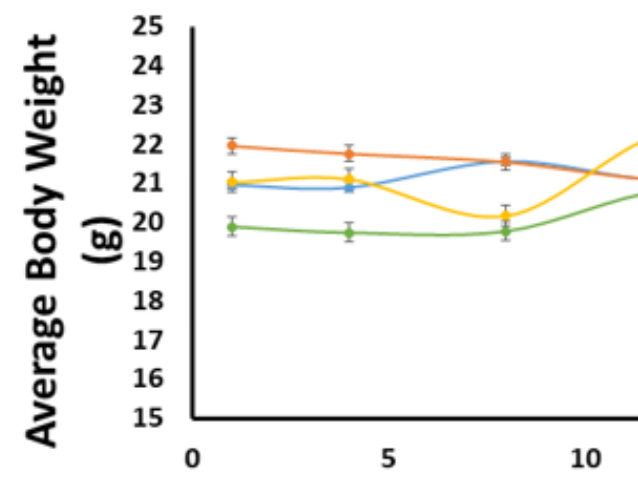

(B)
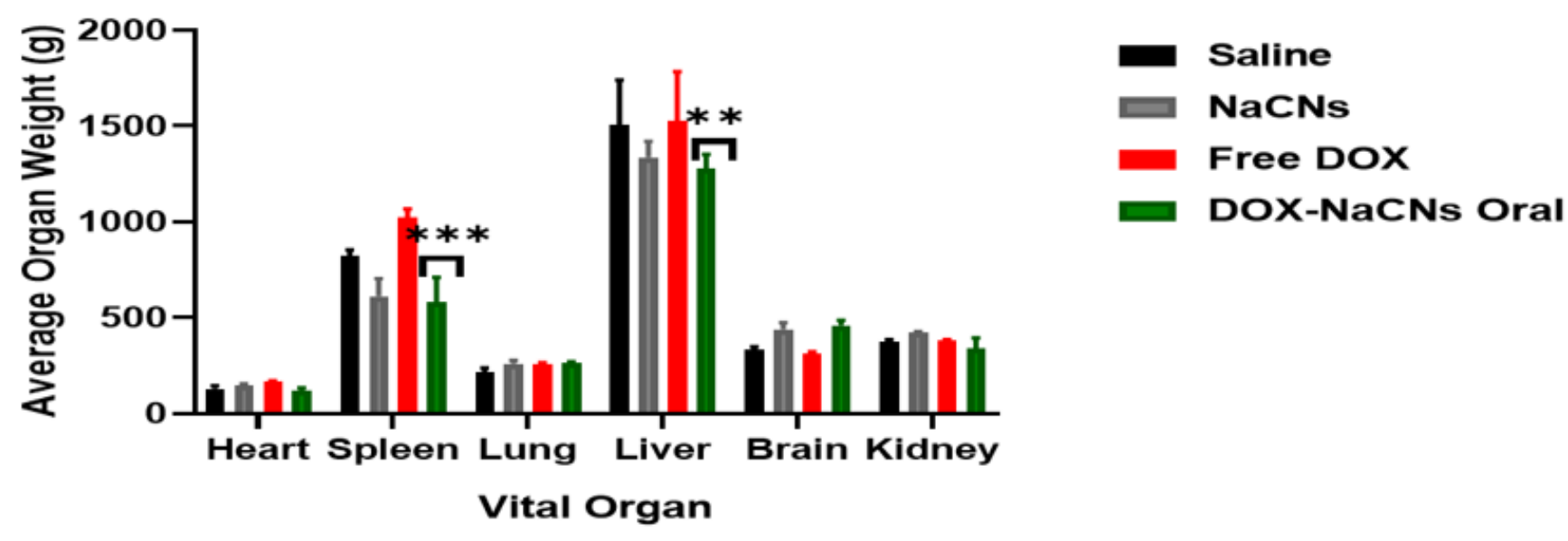

Figure 13 
Average body weight and weight of the vital organs of mice treated orally with saline, NaCNs, free DOX and DOXNaCNs. (A) Average body weight of tumour-induced mice treated orally with NaCNs and DOX-NaCNs when compared with the free DOX and saline-treated group over the 28 days treatment period. (B) The average weight of the vital organs of the negative control, NaCNS, free DOX and DOX-NaCNs treated gro $\neg$ ups on day 28 after the mice were sacrificed, where values are very significant $(* \star)$ at $p<0.01$ and highly significant $(* \star \star)$ at $p<0.001$ vs free DOX-treated groups.
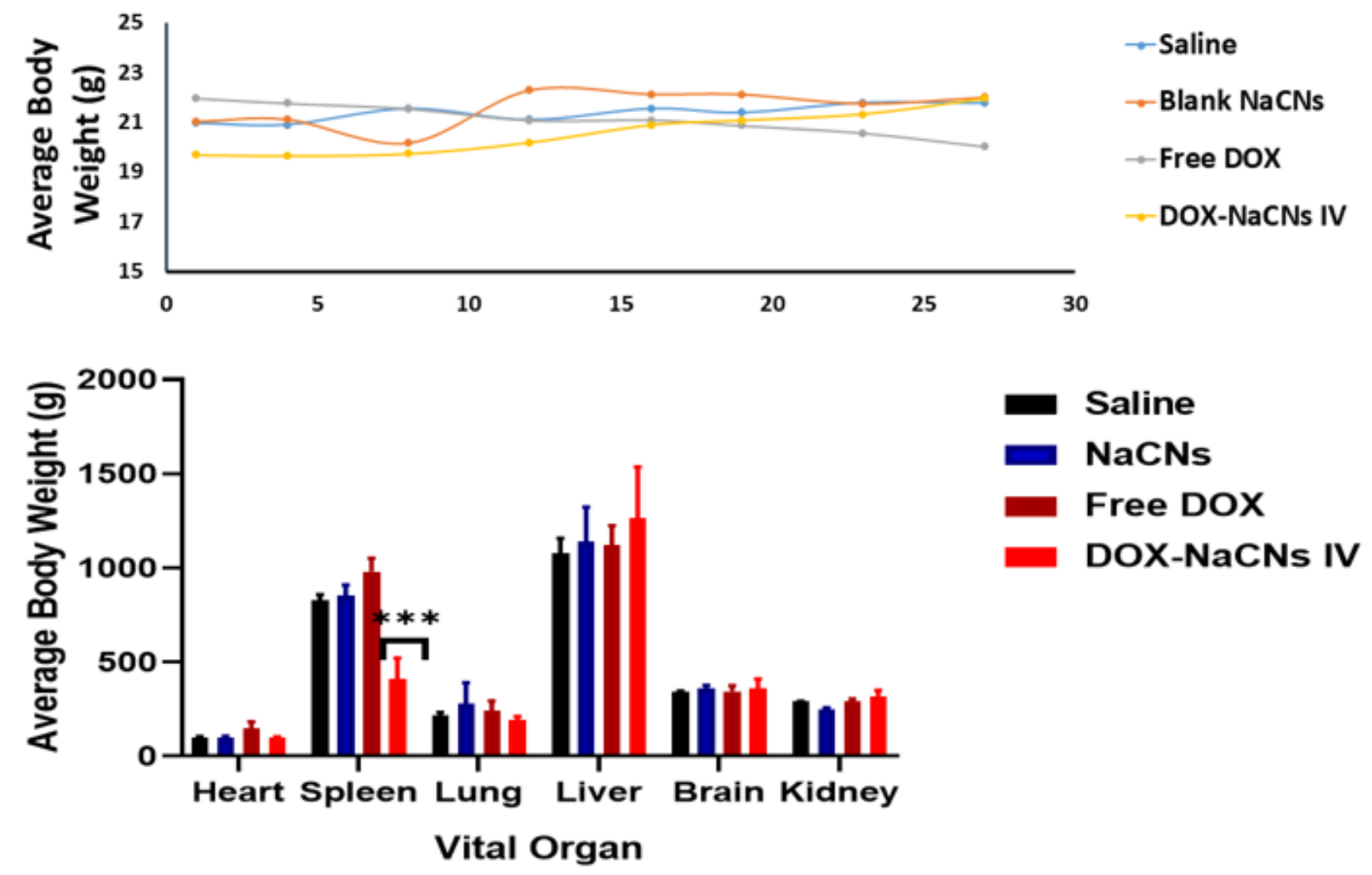

Figure 14

Average body weight and weight of the vital organs of mice treated intravenously with saline, NaCNs, free DOX and DOX-NaCNs. (A) Average body weight of tumour-induced mice treated with intravenously administered NaCNs and DOX-NaCNs when compared with the Free DOX and Saline-treated group over the 28 days treatment period. (B) Average weight of the vital organs of negative control, NaCNS, free DOX and DOX-NaCNs intravenously treated groups on day 28 after the mice were sacrificed, where values are very significant $(* \star)$ at $p<0.01$ and highly significant $(\star \star \star)$ at $p<0.001$ vs free DOX-treated groups. 


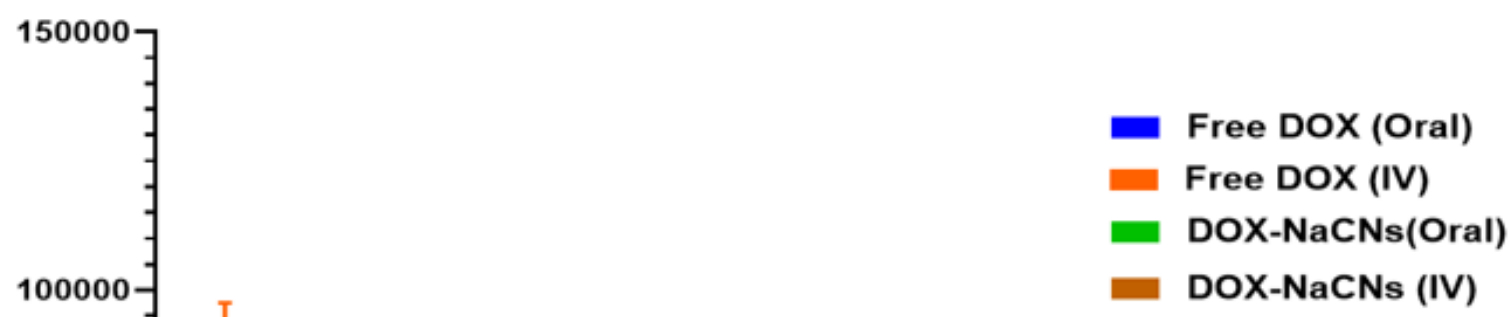

Figure 15

Tumour drug distribution comparison of the orally administered DOX-NaCNs with intravenously administered DOX$\mathrm{NaCNs}$. In vivo biodistribution of both intravenously and orally treated with free DOX and DOX-NaCNs at an equivalent dose of $5 \mathrm{mg} / \mathrm{kg}$ inside mice $(\mathrm{n}=4)$ bearing a xenograft 4T1 tumour. Valued represented biodistribution of both intravenously and orally treated with free DOX and DOX-NaCNs at $24 \mathrm{~h}$ after treatment. Data was shown as mean \pm SD where $n=4$ and \# $p<0.001$ vs Free DOX (IV), @ $p<0.001$ vs DOX- NaCNs (IV), ${ }^{\wedge}<<0.001$ vs Free DOX (oral). 


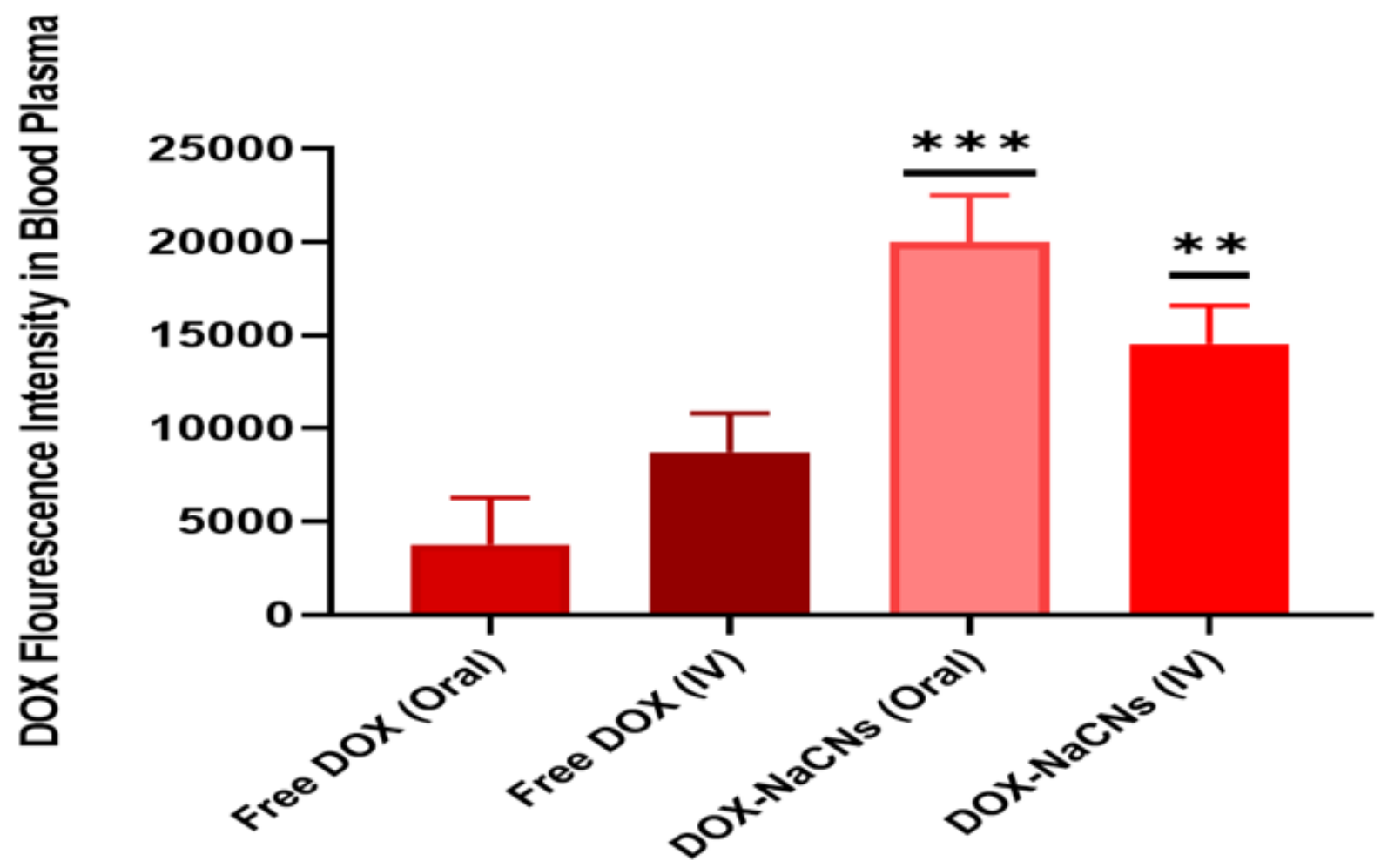

Figure 16

The DOX fluorescence intensity in the blood plasma of Balb/c 4T1 tumour-bearing mice after 24 hours of both intravenous and oral treatments with DOX-NaCNs when compared to free DOX following an intravenous injection. Values were considered very significant $(\star \star)$ at $p<0.01$ and highly significant $(\star \star \star \star)$ at $p<0.001$ and vs. the same treatment with free $\mathrm{DOX}$ at a $\mathrm{Cl}$ of $95 \%$ of representative samples. Values were blank-corrected with the control group.

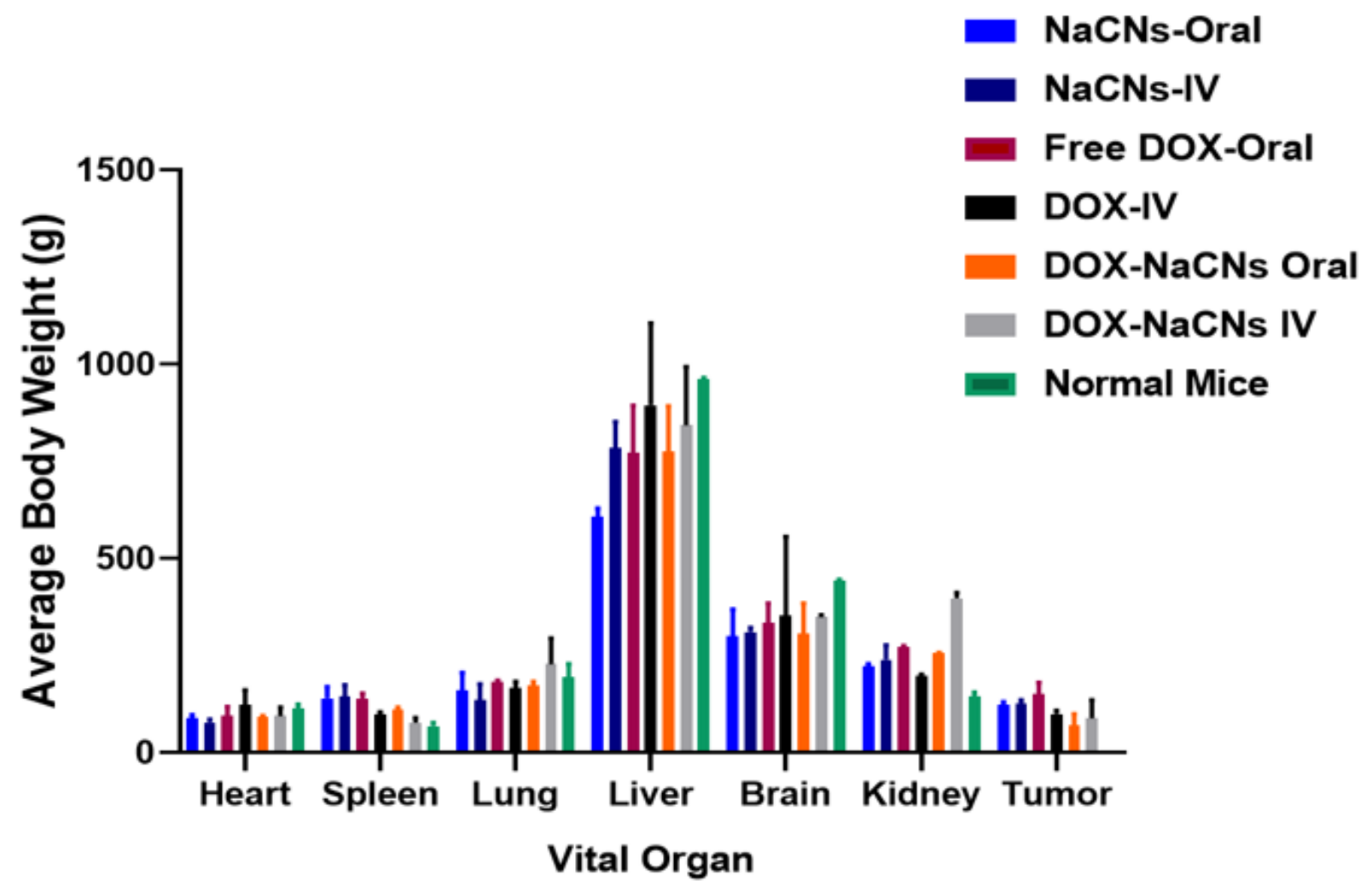


Organ's weight of mice treated intravenously and/or orally with DOX-NaCNs. The average organs weight of tumourinduced mice treated intravenously and/or orally with NaCNs and DOX-NaCNs were compared with the free DOX, control (NaCNs) and normal mice during the biodistribution studies.

(A)

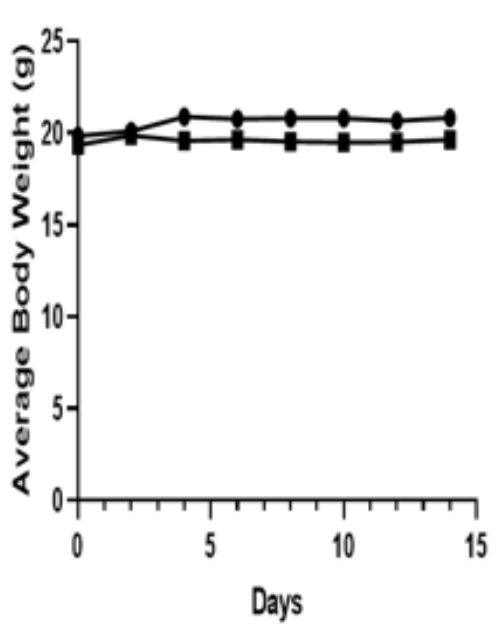

(C)

(D)
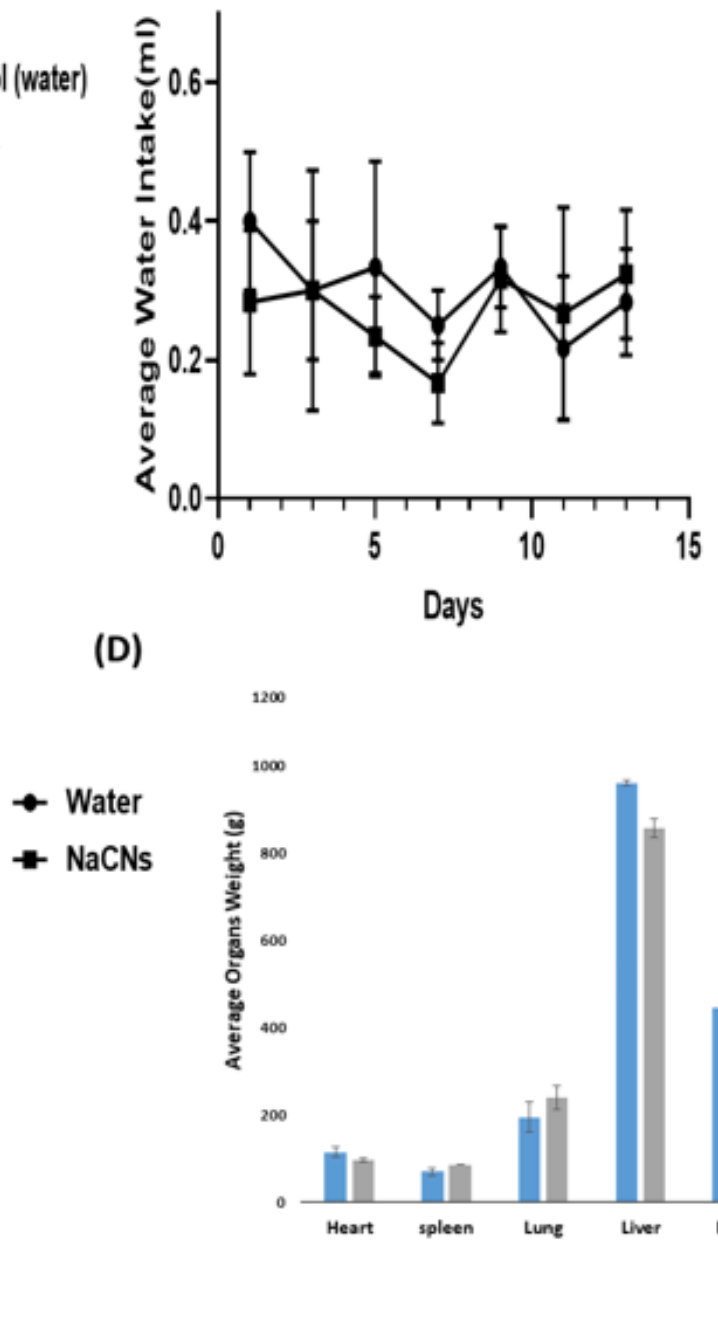

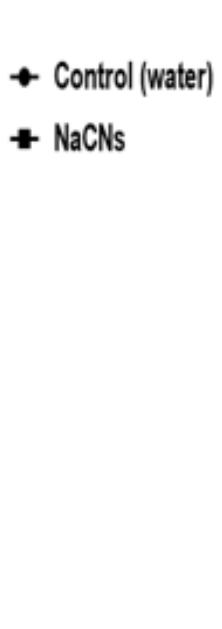

(B)

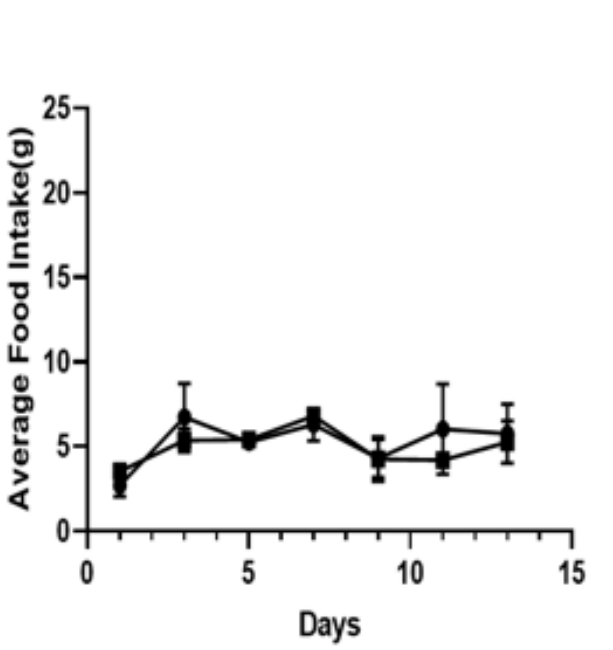

Control (water)

$\mathrm{NaCNs}$
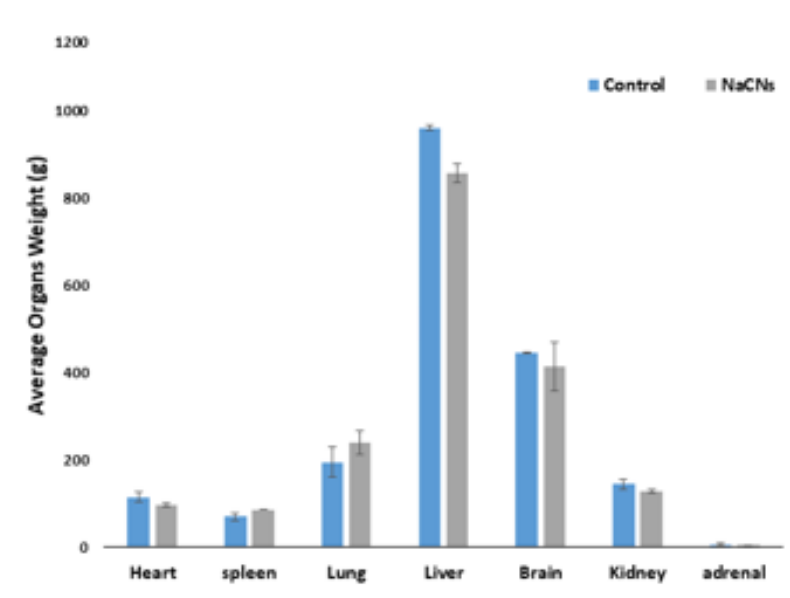

\section{Figure 18}

Bodyweight, water and food consumption analysis during toxicity studies (A) Average body weight of mice (B) Water intake (C) Food intake and (D) Average organ weight for mice during the two-week observation period following NaCNs treatment where group 1, control (\&); group 2, single oral dose of 2,000 mg of NaCNs per kg of body weight. The mean values are shown for five animals/group, with bars indicating the \pm S.D. (standard deviation) and analysed by t-test $(p<0.05)$. The absence of bars means that the \pm S.D. was less than the size of the symbol.

\section{Supplementary Files}

This is a list of supplementary files associated with this preprint. Click to download.

- Graphicalabstract.png 
- SupplementaryMaterials.docx 